\title{
Three-dimensional microenvironment regulates gene expression, function, and tight junction dynamics of iPSC-derived blood-brain barrier microvessels
}

Raleigh M. Linville, ${ }^{1,2,}{ }^{*}$ Matthew B. Sklar, ${ }^{1}$ Gabrielle N. Grifno,,${ }^{1,2}$ Renée F. Nerenberg, ${ }^{1,2}$ Justin Zhou, ${ }^{1,2}$ Robert Ye, ${ }^{1}$ Jackson G. DeStefano, ${ }^{1,3}$ Zhaobin Guo, ${ }^{1}$ Ria Jha, ${ }^{1,2}$ John J. Jamieson,,${ }^{1,4}$ Nan Zhao, ${ }^{1}$ and Peter C. Searson ${ }^{1,2,3, *}$

${ }^{1}$ Institute for Nanobiotechnology, Johns Hopkins University, Baltimore, MD, USA

${ }^{2}$ Department of Biomedical Engineering, Johns Hopkins University, Baltimore, MD, USA

${ }^{3}$ Department of Materials Science and Engineering, Johns Hopkins University, Baltimore, MD, USA

${ }^{4}$ Department of Chemical and Biomolecular Engineering, Johns Hopkins University, Baltimore, MD, USA

*Correspondence: searson@jhu.edu,raleigh@jhu.edu

\begin{abstract}
The blood-brain barrier (BBB) plays a pivotal role in brain health and disease. In the $\mathrm{BBB}$, brain microvascular endothelial cells (BMECs) are connected by tight junctions which regulate paracellular transport, and express specialized transporter systems which regulate transcellular transport. However, existing in vitro models of the BBB display variable physiological accuracy across a wide range of characteristics including gene/protein expression and barrier function. Here, we use an isogenic family of fluorescently-labeled iPSC-derived BMEC-like cells (iBMECs) and brain pericyte-like cells (iPCs) within two-dimensional confluent monolayers (2D) and three-dimensional (3D) tissue-engineered microvessels to explore how 3D microenvironment regulates gene expression and function of the in vitro $\mathrm{BBB}$. We show that 3D microenvironment (shear stress, cell-ECM interactions, and cylindrical geometry) increases BBB phenotype and endothelial identity, and alters angiogenic and cytokine responses in synergy with pericyte co-culture. Tissue-engineered microvessels incorporating junction-labeled iBMECs enable study of the real-time dynamics of tight junctions during homeostasis and in response to physical and chemical perturbations.
\end{abstract}

Keywords (5): blood-brain barrier, induced pluripotent stem cells, brain microvascular endothelial cells, three-dimensional models, gene expression 


\section{Introduction}

Blood vessels in the brain are formed by brain microvascular endothelial cells (BMECs) along with zonation-specific supporting cells. Together these cellular components constitute the blood-brain barrier (BBB) and regulate transport into and out of the brain. BMECs possess intrinsic barrier properties to regulate transport, including enriched expression of: (1) tight junctions (TJs) which block paracellular transport (e.g. claudin-5, occludin, ZO1), (2) efflux pumps which limit passive transcellular transport (e.g. P-gp, BCRP), (3) transporter systems which regulate transcellular nutrient transport (e.g. GLUT-1, Lat1), and (4) specialized fatty acid transporters which restrict vesicular transcytosis (e.g. Mfsd2a) [1]. Collectively these functions limit the passage of $\sim 98 \%$ of small molecules and $\sim 100 \%$ of large molecules into the healthy brain [2], representing a major hurdle for treatment of neurological diseases. Strategies to bypass the BBB for drug delivery include transient tight junction disruption by hyperosmotic agents or focused ultrasound, efflux pump inhibition to increase substrate permeability, and trojan-horse approaches that hijack receptor-mediated transporter systems [3]. While transient loss of barrier function may facilitate treatment of diseased neurons or cancerous cells, permanent changes in barrier function can occur during neurological disease resulting in positive feedback to disease progression [4]. Protection against, or repair of, the different modes of BBB dysfunction is a promising therapeutic strategy to limit neuronal damage during many diseases.

While animal models are indispensable for studies of the BBB, there are considerable species-tospecies variations and technical limitations [5,6], which can be at least partially addressed using in vitro BBB models. However, the lack of appropriate cell sources remains a major obstacle to their development [7-9]. Primary and immortalized BMECs have established endothelial origin, but the permeability of various solutes is much higher than values reported in human and animal studies, and hence physiological barrier function is rarely achieved $[1,10]$. Similarly, transendothelial electrical resistance (TEER), a measure of barrier integrity, has predicted in vivo values of 4,000-8,000 $\Omega \mathrm{cm}^{2}[11,12]$, but is usually less than $200 \Omega \mathrm{cm}^{2}$ for primary and immortalized BMECs [13-16]. Primary and immortalized cell sources have other disadvantages including batch-to-batch variability [13], loss of phenotype during in vitro culture [17], and complicated isolation procedures which limit scalability [5]. To address these limitations, a diverse array of differentiation schemes have emerged to generate BMEC-like cells from induced pluripotent stem cells (iBMECs) (summarized in [18]). These protocols rely on endothelial/mesodermal specification followed by brain endothelial specification, achieved via a combination of Wnt/ $\beta$-catenin, VEGF, and retinoic acid-induced signaling [19-23]. While recent evidence 
suggests that iBMECs possess mixed endothelial and epithelial identity [24], these cells remain a critical source for BBB modeling as they exhibit functional barrier characteristics including high TEER (consistent with physiological measurements), low permeability, efflux activity, and nutrient transport, while enabling highly scalable and patient-specific experimentation [1,18]. Recent approaches aim to drive brain-specificity or endothelial identity by transcription factor (TF) reprogramming of iPSC-derived endothelial cells (iECs) and iBMECs $[24,25]$. While these approaches hold promise, to date they are unable to generate BMECs possessing physiological barrier properties (TEER values typically $<200 \Omega$ $\left.\mathrm{cm}^{2}\right)$.

Assessment of in vitro BBB models has largely relied on measurements conducted in two-dimensional (2D) confluent monolayers which: (1) do not incorporate microenvironmental stimuli present within the native cerebrovasculature, and (2) display limited spatial and temporal resolution. For example, measurements of permeability across 2D confluent monolayers in transwells do not incorporate shear stress and are unable to identify spatial heterogeneity. Microfluidic chip models can incorporate some microenvironmental stimuli (e.g. shear stress), and provide a platform for relatively high-throughput measurements [26, 27].

Here, we differentiated iBMECs from an isogenic family of fluorescently-labeled iPSCs to enable visualization of tight junctions, cytoskeleton, or cell membranes using live-cell imaging in a threedimensional (3D) tissue-engineered BBB model. The two major objective were: (1) to characterize changes in $\mathrm{BBB}$ phenotype caused by 3D microenvironment, and (2) to visualize the dynamics of tight junctions during homeostasis and injury. The 3D microenvironment, which includes shear stress, cellECM interactions, and cylindrical geometry, alters gene expression, protein expression, and functional properties. We addressed conflicting reports of angiogenic and cytokine responses in iBMEC models (in synergy with pericyte co-culture), and showed that the 3D microenvironment alters iBMEC gene expression driving endothelial identity and transcriptomic similarity to human BMECs. Comparison to isogenic non-brain-specific iECs, showed that iBMECs achieve distinct paracellular barrier. To highlight the applications of iBMEC models, we mapped the dynamics of tight junctions during homeostasis, physical injury, and chemical injury. The responses of iBMEC monolayers were found to be unique in 3D microenvironment and across perturbation states (ablation, oxidative stress, and peptide exposure). iBMEC microvessels provide a versatile tool for studying TJ organization, turnover and disruption, critical processes involved in brain health and disease. 


\section{Results}

Differentiation and characterization of fluorescently-labeled isogenic iBMECs

To enable live-cell imaging studies of the BBB during homeostasis and in response to physical, chemical, and cellular perturbations, we developed an isogenic family of BMEC-like cells from CRISPR-edited source iPSCs (iBMECs). We reproduced iBMEC differentiation protocols [20,23] across three isogenic cell lines with fluorescently-labeled ZO1 (iBMEC-TJs), plasma membrane (iBMEC-PMs), and $\beta$-actin (iBMEC-ACTBs) (Fig. S1A). Following differentiation, iBMECs were sub-cultured on collagen IV and fibronectin-coated plates where we observed cobblestone morphology and the formation of confluent monolayers enabling real-time imaging of tight junctions, plasma membrane, and actin cytoskeleton (Fig. S1B). Motivated by a desire to minimize reagent use while maintaining cellular fidelity, we differentiated iBMECs using $1 \mathrm{~mL}$ or $2 \mathrm{~mL}$ of medium to determine impact of media volume on cell phenotype (Fig. S1C). Previous studies have reported the influence of numerous variables on iBMEC differentiation, including hypoxia [27], intermediate mesodermal induction [22, 28], media composition [29], serum free alternatives [30], source/choice of ECM coating material [31-33], and iPSC seeding density [34]. With reduced media volume during differentiation, iBMECs displayed improved phenotype as measured by TEER, paracellular and transcellular permeability, protein expression, and gene expression (see Note S1 and Fig. S2). Reduced media volume increased the fraction of all singularized cells which attached to sub-culture plates $(p=0.023)$, suggesting increased purity of endothelial-like cells resulting from the differentiation (Fig. S1D). Reduced media volume also increased the barrier function of iBMEC monolayers when cultured on transwells, with $\sim 2$-fold higher average TEER across all cell lines and time points (Fig. S1E, S2A, B). Mean TEER values for all cell lines and differentiations were within the range of in vivo measurements in animal models and theoretical calculations $\left(1,500-8,000 \Omega \mathrm{cm}^{2}\right)$ [1]. Reduced media volume lowered Lucifer yellow (444 Da) permeability measured two days after culturing iBMECs onto transwells ( $p=0.043$ ) (Fig. S2C), while not substantially altering the permeability of $10 \mathrm{kDa}$ dextran $(p=0.288)$ (Fig. S2D) or the directional transport of glucose $(p=0.353)$ or rhodamine $123(p=0.054)$ (Fig. S2E). Gene and protein expression of endothelial and BMEC markers were similar regardless of media volume (Fig. S2F-H). Together these results show that reduced media volume can be used to differentiate iBMECs that display functional hallmarks of human BMECs. We explored variability of iBMEC differentiation (following cryopreservation, using an independent serum lot, and using an independent iPSC source) and the relationship between TEER and permeability across our datasets (see Note $\mathbf{S} 2$ and Fig. S3). Additionally, the mechanistic underpinnings of the reduced media volume effect 
remain to be fully elucidated, but are likely related to soluble factors secreted during the initial UM/Fphase of differentiation (see Note S3 and Fig. S4).

\section{Barrier function of $3 D$ iBMEC microvessels}

Tissue-engineered microvessels were generated by seeding iBMECs into $150 \mu \mathrm{m}$ diameter channels patterned in collagen I (Fig. 1A-i). iBMECs formed confluent $\sim 1 \mathrm{~cm}$-long microvessels free from defects as evidenced by a lack of $10 \mathrm{kDa}$ dextran leakage (Fig. 1A-ii). The library of fluorescently-labeled isogenic iPSCs enabled real-time imaging of tight junctions, plasma membrane, and actin cytoskeleton via epifluorescence or confocal microscopy (Fig. 1B). iBMEC microvessels maintained low Lucifer

yellow (444 Da) permeability, with no visible sites of paracellular leakage indicating formation of a confluent monolayer connected by tight junctions (Fig. 1C). Across the three cell lines there were highly similar Lucifer yellow permeabilities, with an average value of $\sim 2.3 \times 10^{-7} \mathrm{~cm} \mathrm{~s}^{-1}$. Compared to induced isogenic endothelial cell (iEC) microvessels, iBMEC microvessels displayed $\sim 60$-fold lower permeability demonstrating acquisition of brain-specific barrier properties (Fig. 1D). For both iBMECs and iECs, 3D culture was associated with reduced paracellular permeability $(p<0.0001$ and $p=0.0010$, respectively) (Fig. 1D).

In 2D, the permeability of iBMEC monolayers increased $\sim 6$-fold over six days $(p=0.078$ ) (Fig. 1E, $\mathrm{S} 2 \mathrm{C}$ ). In contrast, 3D iBMEC microvessels maintained stable barrier function in the absence of cocultured cells over at least six days $(p>0$.9240) (Fig. 1E). The incorporation of iBMEC-PMs into iBMEC microvessels enabled identification of the luminally localized glycocalyx and exclusion of $10 \mathrm{kDa}$ dextran from the endothelium (Fig. 1F). These results suggest that the $3 \mathrm{D}$ microenvironment promotes physiological and stable paracellular barrier function. 


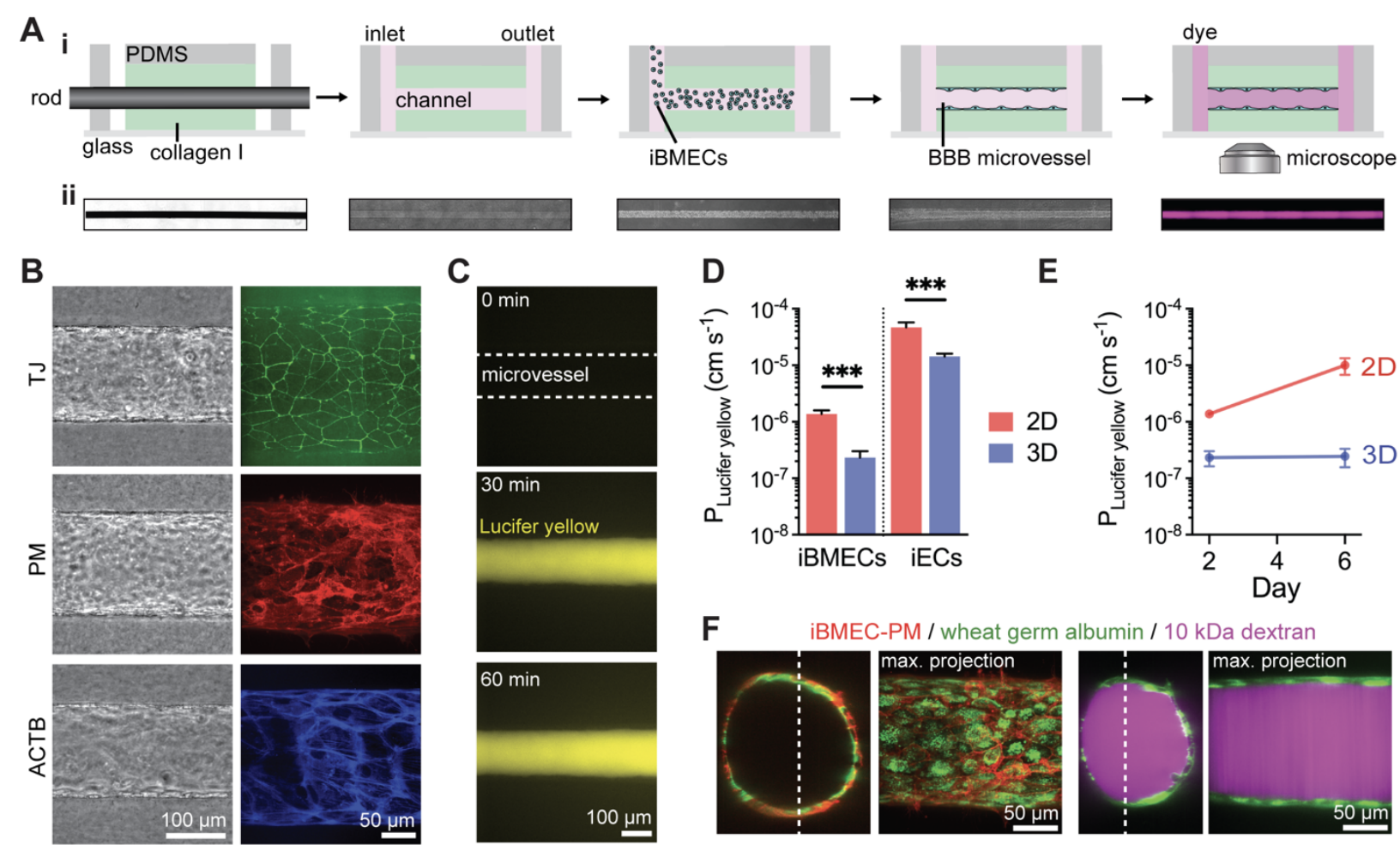

Figure 1. Three-dimensional BBB model incorporating iBMECs displays stable and low paracellular permeability.

(A) Fabrication and imaging of iBMEC microvessels. $150 \mu \mathrm{m}$ diameter channels patterned in collagen I were seeded with iBMECs to form a confluent endothelium. Insets below show phase contrast and fluorescence images along the experimental timeline.

(B) Representative images show organization of iBMEC microvessels with localized TJ, PM, and ACTB expression under confocal imaging (max. intensity projection shown).

(C) Representative images following perfusion with Lucifer yellow.

(D) Permeability of Lucifer yellow on day 2 across microvessel conditions: iBMEC microvessels $(n=$ 14), iBMEC transwells $(n=11)$, isogenic iEC microvessels $(n=4$ microvessels $)$, and iEC transwells $(n=$ 4).

(E) Time course of Lucifer yellow permeability over one week for iBMEC microvessels $(n=14$ and 4 , respectively) and iBMEC transwells $(n=11)$.

(F) Confocal images of iBMEC-PM microvessels highlighting the glycocalyx and showing solute perfusion in the lumen. (Left) Wheat germ albumin staining of iBMEC glycocalyx. (Right) $10 \mathrm{kDa}$ dextran not observed to accumulate within glycocalyx or iBMECs (60 minutes after exposure).

Data are presented as mean \pm SEM. $* * * p<0.001$. See also Figure S1-4. 


\section{Gene expression of $3 D$ iBMEC microvessels}

To facilitate comparison of iBMECs to other EC and BMEC datasets, we conducted meta-analysis of global gene expression collected across several bulk RNA-sequencing studies (Fig. 2A). We performed in-house sequencing of iBMECs in 2D monolayers and 3D microvessels, primary human dermal microvascular endothelial cells (HDMECs), iPSC-derived endothelial cells (iECs) generated by transient expression of ETV2 after mesodermal induction [35], and neural crest iPSC-derived pericytes (iPCs) generated using published protocols [36]. Data for HDMECs, iECs, and iPSCs were obtained from 2D confluent monolayers. Previously published RNA transcriptomes were obtained from the NCBI Gene Expression Omnibus (GEO) from the following studies: (1) iBMECs studied in a 2D microfluidic chip model to probe the influence of shear stress [26], (2) immunopanned human BMECs [37], (3) isolated human brain microvessels [5], (4) iBMECs reprogrammed using ETS transcription factors [24], (5) iECs treated with RepSox to induce barrier properties [38], and (6) immortalized BMECs [39] (Table S1). Principal component and Spearman correlation analysis identified similarities between immunopanned human BMECs and human brain microvessel gene profiles, with separate clusters of iBMEC samples and other endothelial cell samples (Fig. 2A, B). iBMECs and other EC samples displayed unique gene expression profiles compared to human BMEC samples with Spearman correlation coefficients $(\rho)$ ranging from 0.72 to 0.77 . The group of cells including immortalized BMECs, iECs treated with RepSox, iBMECs reprogrammed using ETS transcription factors, and iECs generated in this study by transient expression of ETV2, were similar to each other $(\rho=0.89$ to 0.94$)$. However, none of these cell sources exhibited TEER values resembling in vivo measurements [1]. In contrast, iBMECs had unique gene expression profiles compared to the group of endothelial cells suggesting a reduction in endothelial identity [24], but exhibited physiologically high TEER. The 3D microenvironment slightly increased the correlation of iBMECs to immunopanned human BMECs and human brain microvessels (average 1\% increase), while this effect was not observed for iBMECs cultured under shear stress alone (average 1\% decrease) (Fig. 2B), suggesting that other components of the 3D microenvironment may be important in promoting brain identity [26]. 

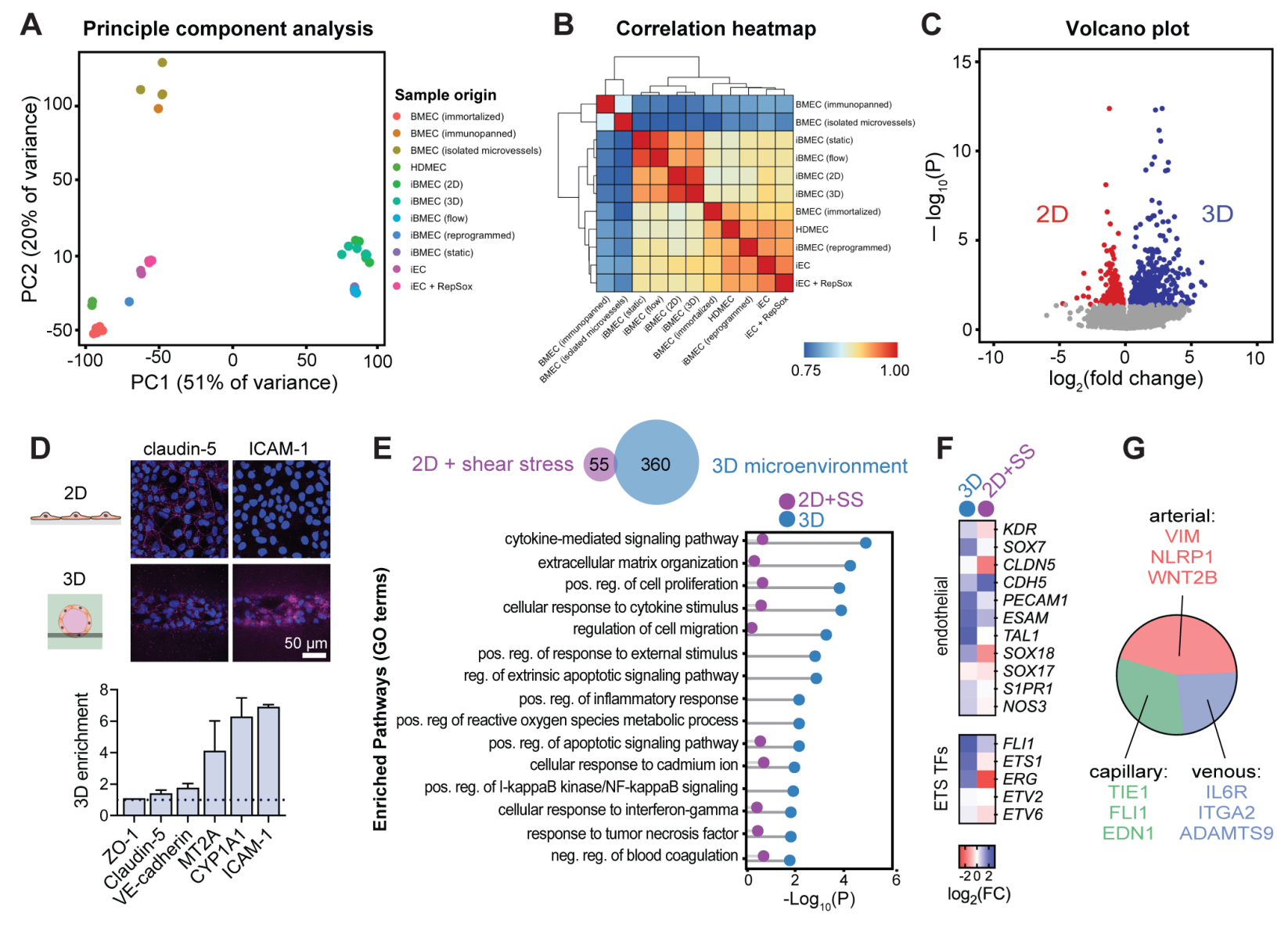

Figure 2. Three-dimensional microenvironment regulates iBMEC gene expression.

(A) Principal component analysis (PCA) of iBMECs, endothelial cells, and human tissue datasets across environmental conditions. Data includes iBMECs in 2D and 3D across two cell lines $(n=5)$, iECs in 2D $(n=2)$, among samples across other studies summarized in Table S1.

(B) Heatmap of Spearman correlation coefficients across studies. iBMECs, endothelial cells, and human BMEC datasets cluster independently.

(C) Volcano plots depicting significantly (adjusted $p<0.05$, Wald test with Benjamini-Hochberg correction) upregulated genes (blue) and downregulated genes (red) in iBMECs in 3D microvessels and 2D monolayers $(n=5)$.

(D) Semi-quantitative validation of protein enrichment in 3D microvessels. Representative immunofluorescence images from $n=2$ biological replicates for claudin-5 and ICAM-1 in 2D and 3D are shown. Data is normalized to nuclei signal and endothelium area and presented as mean \pm SEM.

(E) Comparison of upregulated genes between 3D microenvironment and 2D monolayers under shear stress (previously published dataset (ref. 26)). Genes represented in Venn diagram and Lollipop plots are differentially expressed genes (DEGs) upregulated by 3D microenvironment and shear stress by $\log _{2} \mathrm{FC}>$ 1. Venn diagram shows overlap of genes between studies, while Lollipop plot highlights select pathways (GO biological process terms).

(F) Heatmap of gene enrichment by 3D microenvironment versus shear stress for endothelial genes and ETS transcription factors. 
(G) 3D microenvironment DEGs are associated with zonation specific human BMEC gene expression $(\sim 10 \%)$, where arterial, capillary, and venous identity are equally represented. Three example zonationspecific genes upregulated by 3D microenvironment are shown for each zone.

See also Figure S5 and Data S1.

\section{$3 D$ microenvironmental regulation of gene expression}

Bulk RNA sequencing of iBMECs across two independent iPSC sources in 3D microvessels revealed 497 upregulated genes and 228 downregulated genes compared to iBMECs in 2D static monolayers $(\rho=0.975)$

(Fig. 2C, Data S1). We validated upregulation of several genes at the protein level using immunocytochemistry. Metallothionein-2 (MT2A), a cytochrome P450 super family member (CYP1A1), and ICAM-1 (ICAMI) were confirmed to be enriched 4 -6-fold in 3D microvessels based on semiquantitative analysis of relative protein expression normalized to the fluorescence of DAPI-stained nuclei (Fig. 2D). CYPIA1 and MT2A expression had previously been found to be shear-stress dependent in primary or iPSC-derived BMECs [26, 40-42]. We confirmed that the tight junction proteins claudin-5 (CLDN5) and zona occludens-1 (TJP1), which were not substantially upregulated at the gene level by the 3D microenvironment, were also similarly expressed at the protein level (Fig. 2D).

In contrast to studies of the role of laminar flow on 2D monolayers [26, 43], here tissue-engineered iBMEC microvessels were exposed to stimuli including cell-ECM interactions, cylindrical geometry, and transmural pressure. We identified 360 genes upregulated by greater than two-fold compared to only 55 genes upregulated to this same level by 2.4 dyne $\mathrm{cm}^{-2}$ shear stress in a previous study [26]; less than $1 \%$ of upregulated genes were shared, indicating unique modes of gene expression changes (Fig. 2E). To predict functional differences induced by shear stress and 3D microenvironment, we performed pathway enrichment analysis on two-fold-enriched differentially expressed genes compared to static or 2D conditions. Biological processes upregulated by the 3D microenvironment minimally overlapped with those upregulated by shear stress (Data S1). Unique biological processes induced by the 3D microenvironment included GO terms associated with cytokine-mediated signaling pathways, extracellular matrix organization, regulation of cell migration, positive regulation of inflammatory response, and negative regulation of blood coagulation (Fig. 2E). 
Additionally, we found that the 3D microenvironment enhanced the endothelial identity of iBMECs, upregulating endothelial markers and ETS transcription factors that have previously been reported to be attenuated in iBMECs compared to various endothelial cell sources (Fig. 2F) [24]. Shear stress alone resulted in minimal changes in these same genes, but neither shear stress nor 3D microenvironment dramatically altered expression of epithelial genes (data not shown). Lastly, we benchmarked our microvessel model to recently published single nucleus RNA-sequencing of the human cerebrovasculature, finding that $15 \%$ of genes enriched by the 3D microenvironment display zonation specificity in BMECs [44]. These genes are roughly equally distributed across arteriole, capillary, and venule BMEC clusters, indicating that while 3D microenvironment can enrich for genes expressed by the human cerebrovasculature, a zonation-specific identity is not promoted (Fig. 2G).

\section{Microenvironmental regulation of angiogenic and cytokine response}

To explore the synergy between microenvironmental factors regulating iBMEC phenotype, we incorporated iPSC-derived pericytes into our tissue-engineered BBB model. Pericytes exert diverse influences on BMECs, including regulation of angiogenesis and leukocyte infiltration [45-47]. An isogenic model was generated by culturing iPSC-derived neural-crest pericytes (iPCs) in microvessels for one day prior to seeding iBMECs. The iPCs maintained direct cell-cell contact with iBMECs and were located along the abluminal surface of the endothelium (Fig. 3A). iPCs were validated by expression of NG2 and PDGFR $\beta$ at the gene and protein level (Fig. S5C, D). To explore the response to growth factors

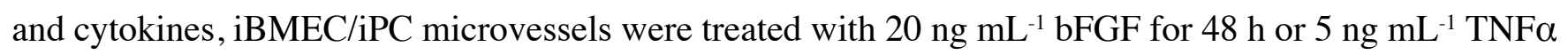
for $24 \mathrm{~h}$ (Fig. 3B).

The angiogenic response was compared across three conditions (microvessels, microvessels cocultured with pericytes, and a bead assay) by measuring the total length of sprouts per unit area of endothelium. As previously observed, iBMECs were responsive to growth factors, including bFGF [48] (Fig. 3C). In the absence of bFGF, iBMEC microvessels displayed no sprouting, while bFGF treatment increased sprout length (Fig. 3D) and resulted in the formation of a visible lumen. iBMEC/PC microvessels displayed elevated responsiveness to bFGF with longer sprout lengths, visible lumens, and pericytes located at the leading edge (Fig. 3E). 

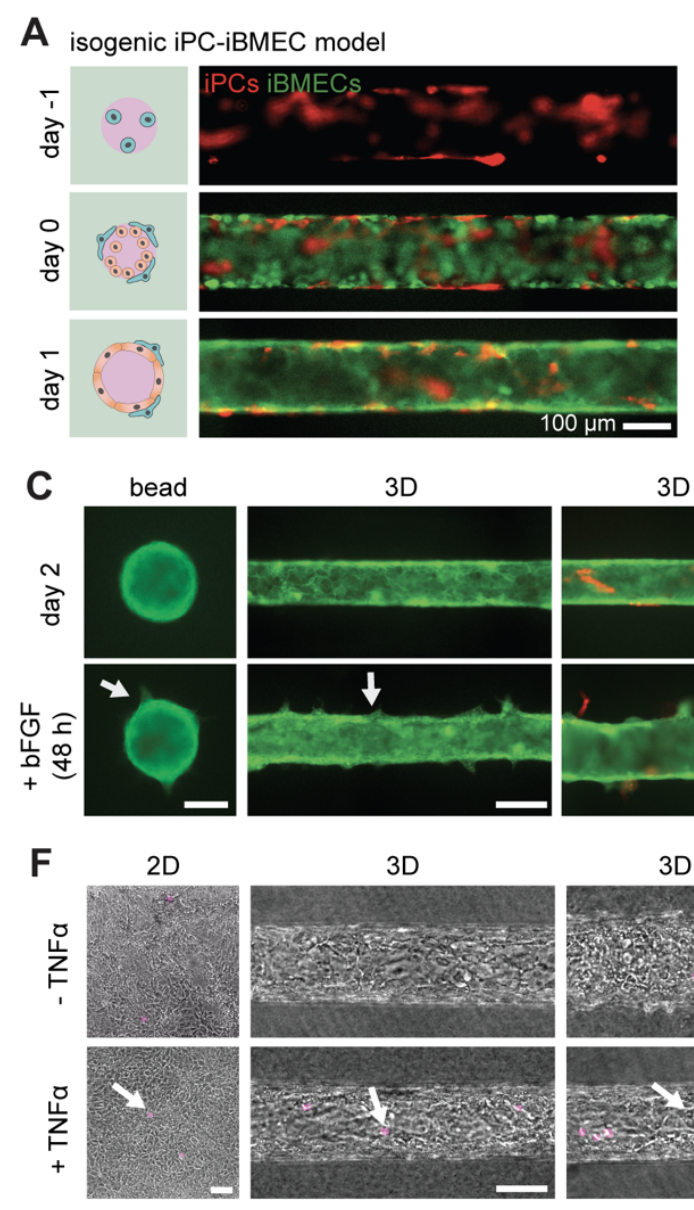

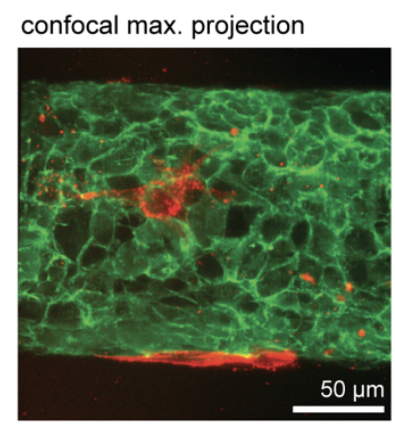

B

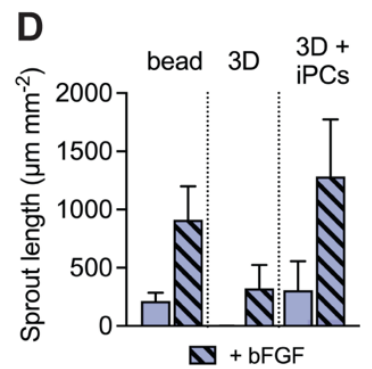

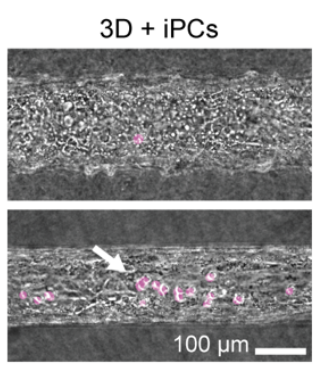
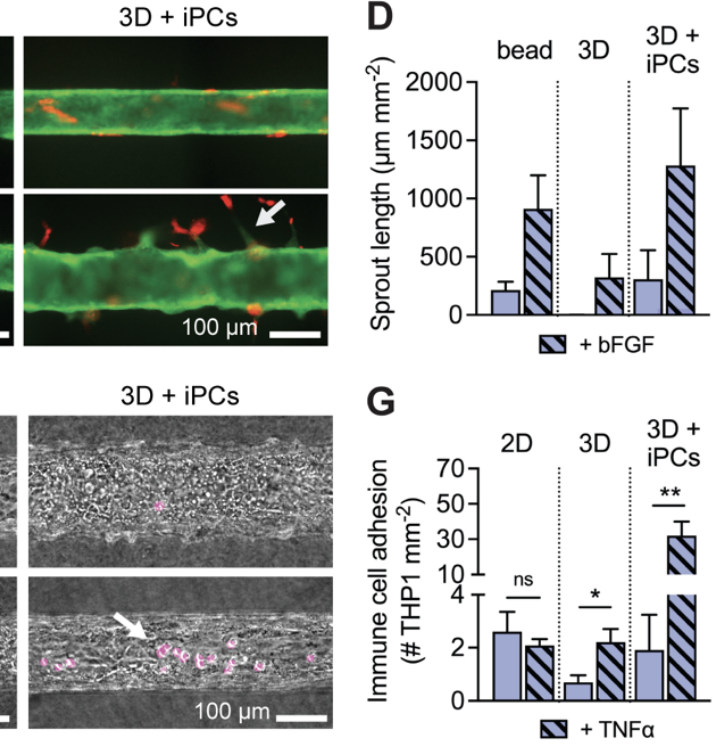
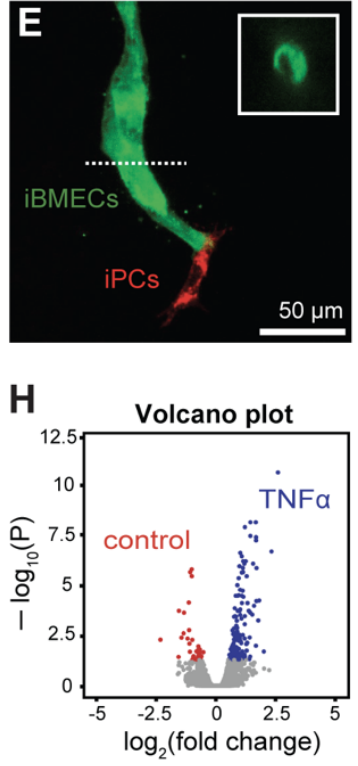

Figure 3. Three-dimensional microenvironment and pericyte co-culture synergistically alter angiogenic and cytokine responses.

(A) The combination of ACTB-labeled iBMECs and PM-labeled iPCs enables creation of an isogenic coculture BBB model. (i) The model was formed via sequential seeding of pericytes and then endothelial cells, to achieve a 1:3 ratio. (ii) Pericytes localized abluminal to iBMEC-PM.

(B) Experimental design: 2D, 3D and 3D models co-cultured with pericytes were exposed to bFGF or $\mathrm{TNF} \alpha$ to quantify inflammatory and angiogenic responses, respectively.

(C) Representative images of iBMEC response to bFGF. Angiogenic sprouts appear over two days of exposure (white arrows).

(D) Quantification of angiogenic sprout length following $48 \mathrm{~h}$ exposure to $20 \mathrm{ng} \mathrm{mL}^{-1} \mathrm{bFGF}$. Data collected across bead assay $(n=4), 3 \mathrm{D}$ microvessels $(n=4)$, and pericyte co-cultured 3D microvessels $(n$ $=3$ ).

(E) Pericytes located at the leading edge of an angiogenic sprout. Inset: representative lumen cross-section at location of dotted line.

(F) Representative images of $\mathrm{iBMEC}$ response to TNF $\alpha$. Experiments were performed on $\mathrm{BC} 1 \mathrm{iBMECs}$ paired with RFP-tagged WTC iPCs to enable detection of adherent immune cells (white arrows).

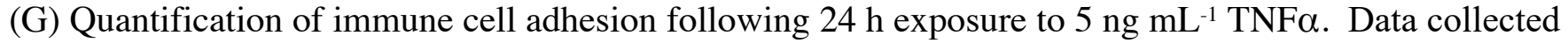
across 2D monolayers $(n=8), 3 \mathrm{D}$ microvessels $(n=5)$, and pericyte co-cultured 3D microvessels $(n=4)$. 
(H) Volcano plots showing significantly (adjusted $p<0.05$, Wald test with Benjamini-Hochberg correction) upregulated genes (blue) and downregulated genes (red) following $24 \mathrm{~h}$ exposure to $5 \mathrm{ng} \mathrm{mL}$ ${ }^{1} \mathrm{TNF} \alpha$ in 3D microvessels ( $n=2$ biological replicates).

Data are presented as mean \pm SEM. $* p<0.05$, ** $p<0.01$, *** $p<0.001$, and $* * * * p<0.0001$. See also Data S2.

Previous studies of iBMECs have reported conflicting degrees of responsiveness to inflammatory cytokines $[22,24,49,50]$. Here, we assessed the response of 2D iBMEC monolayers, microvessels, and iBMEC/iPC microvessels to TNF $\alpha$ by measuring the adherence of monocyte-like cells (THP1s) (Fig. 3F). In 3D, THP1 adhesion was measured under perfusion at low shear stress, while in 2D, media washes were conducted after static exposure to remove non-adherent cells. In 2D monolayers, the density of adhered THP1s was relatively high and did not increase following TNF $\alpha$ exposure $(p=0.5136)$. In 3D, immune cell adhesion was low under control conditions, but increased $\sim 3$-fold following TNF $\alpha$ exposure $(p=$ 0.0259) (Fig. 3G). Furthermore, in the presence of pericyte co-culture immune cell adhesion was dramatically enhanced upon TNF $\alpha$ exposure $(\sim 16$-fold $)(p=0.0094)$. These results suggest that the dynamics of immune cell adhesion are synergistically modulated by the 3D microenvironment and pericytes.

We also compared gene expression of iBMEC microvessels with and without TNF $\alpha$ exposure by bulk RNA sequencing, identifying 136 upregulated genes and 35 downregulated genes $(\rho=0.971)$ (Fig. 3H, Data S2). Upregulated genes included NF- $\mathrm{BB}$ family members (NFKB1, NFKB2), endothelial transmembrane proteins facilitating leukocyte transmigration (ICAMI), cytokines (CCL2, CXCL16), growth factors (PDGFA, PDGFB), cell-cell junctions (CLDN4, TJP2) and integrins (ITGB3, ITGB6), highlighting diverse downstream effects of cytokine exposure on iBMECs.

\section{Dynamics of iBMEC monolayers in tissue-engineered microvessels}

Given the unique phenotype of 3D microvessels, we sought to apply this model to understand BBB behavior during homeostasis and injury. We performed live cell imaging of iBMEC-TJ monolayers in tissue-engineered microvessels to assess tight junction dynamics. ZO1 (TJP1) is a scaffolding protein that connects transmembrane TJ proteins to the actin cytoskeleton [51], enabling live cell measurements of cell morphology, motility, and the number of nearest neighbors (i.e. shared boundaries) (Fig. 4A). Fluorescence imaging revealed that the tight junctions were dynamic, with continuous small fluctuations 
in the position of cell-cell junctions between adjacent cells. Under homeostatic conditions, most cells were quiescent ( $\sim 97 \%$ ) during imaging and displayed highly stable morphology maintaining $\sim 6$ nearest neighbors (Fig. 4B).

In homeostasis, both mitosis and cell loss were observed, although the net monolayer turnover was very low (typically $<0.5 \% \mathrm{~h}^{-1}$ ). Interestingly, while rates of mitosis and turnover were similar between 2D and 3D culture $(p>0.05)$, cell loss rates were elevated in the 3D microenvironment $(p=0.0140)$ (Fig. 4C), potentially related to RNA sequencing results finding upregulation of genes associated with extrinsic apoptotic signaling pathway. Imaging during permeability measurements showed that iBMECs were able to reorganize their junctions to maintain barrier function during apoptosis and mitosis, thereby preventing transient leakage. Cell loss events showed the hallmarks of apoptosis, with visible nuclear fragmentation and formation of apoptotic bodies under phase contrast imaging, and subsequent cell collapse under fluorescence imaging ( $t=0$ defined as end of cell collapse) (Fig. S6A). Mitotic cells were visible based on chromosomal alignment and separation under phase contrast imaging, and cytokinesis under fluorescence imaging ( $t=0$ defined as separation of daughter cells via tight junction) (Fig. S6B).

We recorded the morphology of 7 quiescent, 8 mitotic, and 7 apoptotic cells along with all their nearest neighbors (NN) and progeny ( $n=151$ cells) with 5-minute resolution for 30 minutes before and 30 minutes after these events ( $t=0$ defined above) (Fig. 4D, S7). First, we performed principal component analysis (PCA) of cell morphology (6 morphological metrics of 151 cells imaged over at least 60 minutes). The first four principal components were: (1) cell size (area and perimeter), (2) cell shape (circularity and aspect ratio), and (3) cell motility (instantaneous speed), and (4) number of cell neighbors (local density of cells in a monolayer) (Fig. S6C). Although the number of cell neighbors was highly correlated with cell area, there were distinct contributions of cell neighbors to single cell morphology warranting inclusion as a separate metric. 

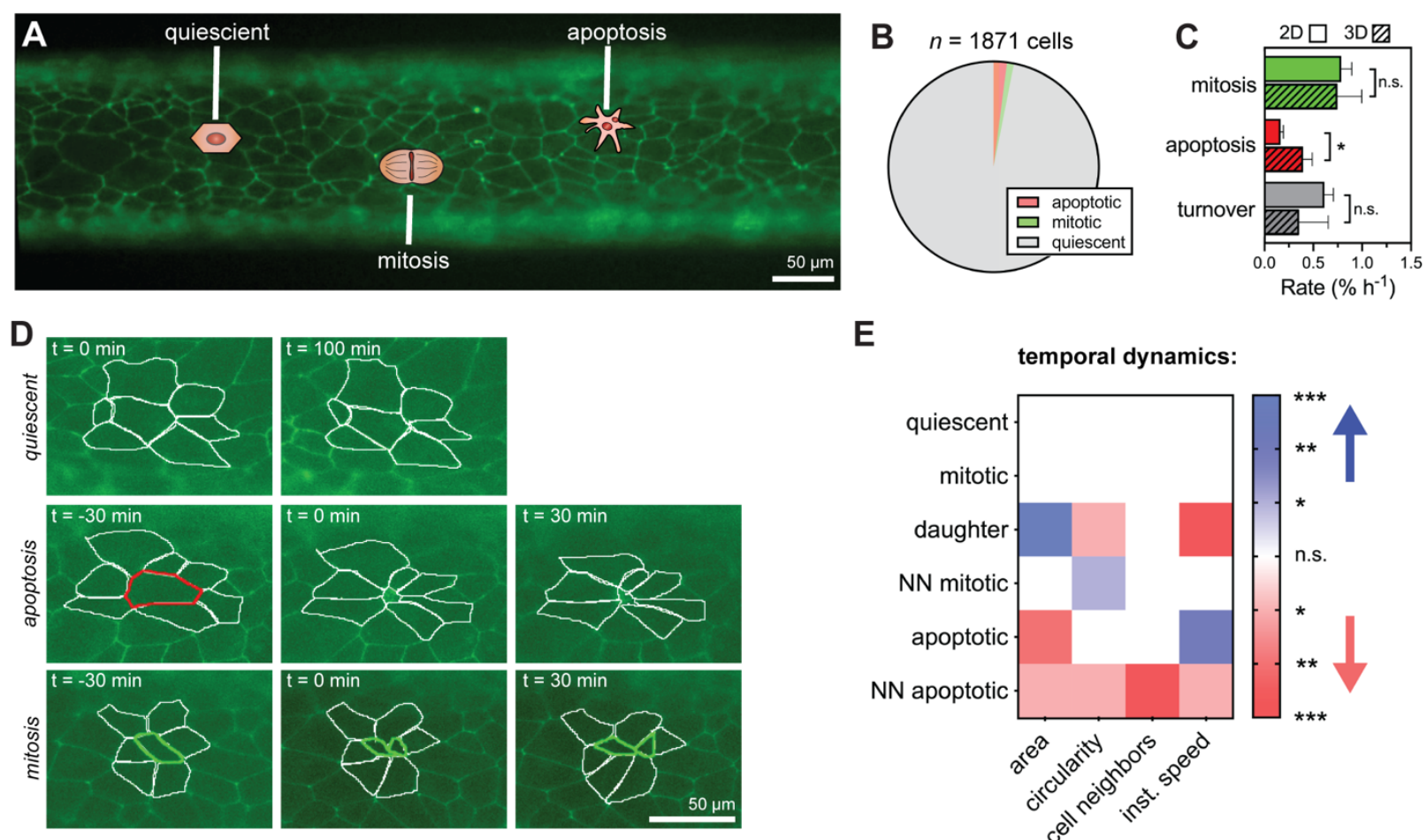

E
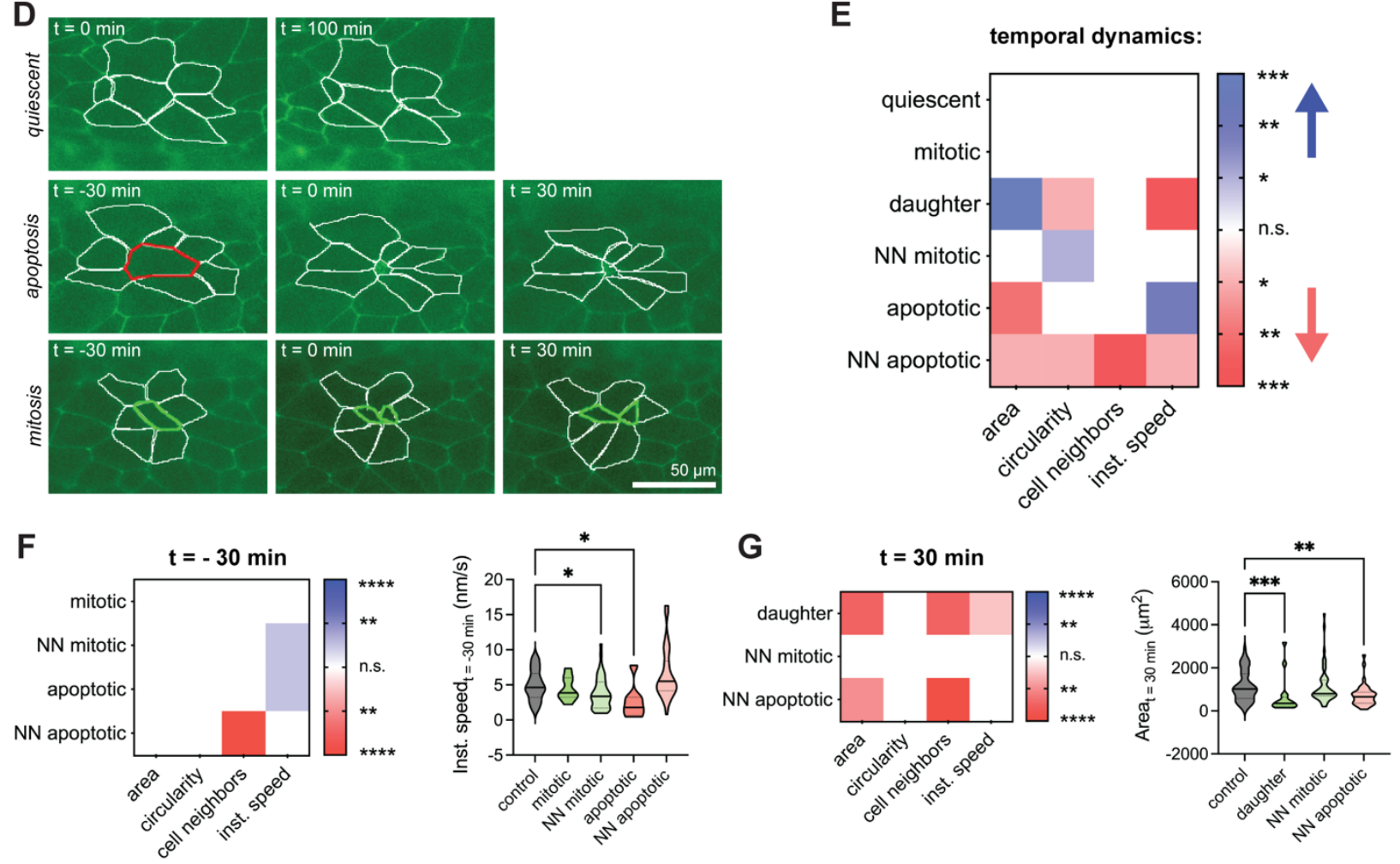

Figure 4. Monolayer dynamics in three-dimensional iBMEC microvessels.

(A) Tight junction-labeled iBMECs support tracking of monolayer dynamics in real time.

(B) Apoptotic, mitotic, and quiescent cell populations identified from imaging of $n=1871$ cells from $n=$ 6 iBMEC-TJ microvessels.

(C) Comparison of mitosis, apoptosis, and turnover rates between iBMECs in 3D microvessels $(n=7)$ and 2D monolayers $(n=6)$. Significance calculated using a Mann-Whitney test.

(D) Apoptotic, mitotic, and quiescent cells are identified from changes in tight junction fluorescence and phase contrast imaging over 100 minutes for quiescent cells and 60 minutes for apoptotic and mitotic cells, with an interval of 5 minutes.

(E) Heatmap summarizing morphological changes during imaging. Color signifies direction of change, while shade signifies level of significance as calculated using the maximally significant result between a Wilcoxon matched-pairs signed rank test to compare first and last time points and using an $F$ test to determine if linear regression of the morphological time course displayed a statistically non-zero slope. $\mathrm{NN}$ - nearest neighbor. 
$(\mathrm{F}, \mathrm{G})$ Heatmaps comparing morphological metrics of cell types before $(\mathrm{t}=-30 \mathrm{~min})$ and after $(\mathrm{t}=30$ min) cellular events. Significance and direction of any difference was calculated using a Kruskal-Wallis test and post hoc Dunn's multiple comparisons test relative to quiescent cells.

$* p<0.05, * * p<0.01$, and $* * * p<0.001$. See also Figure S6-8.

Mitotic cells displayed no changes in morphology prior to formation of daughter cells $(p>0.05$ for all metrics). Daughter cells increased in size following division, while the circularity and instantaneous speed decreased. The nearest neighbors of mitotic cells displayed a decrease in circularity over the 60-minute period encompassing 30 minutes before and after cytokinesis. Apoptotic cells displayed loss of area and perimeter, as well as an increase in instantaneous speed. Interestingly, the nearest neighbors of apoptotic cells displayed the most substantial morphological changes highlighting their important role in maintenance of barrier function during this process. During apoptosis, the nearest neighbors displayed a reduction in area $(p=0.0144)$, showing that they do not contribute to compensation of the loss of monolayer area. This observation implies that more distant cells must increase in area and push the nearest neighbors into the area lost by the apoptotic cell. The extrusion of apoptotic cells has been reported for epithelial monolayers [52]. Additionally, the nearest neighbors of apoptotic cells displayed reduced circularity, number of cell neighbors (as a neighbor was lost by the end of apoptosis), and highly dynamic instantaneous speeds that were increased after the point of cell collapse but reduced at 30 minutes after apoptosis compared to 30 minutes before $(p=0.0117,<0.0001$, and 0.0249 , respectively) (Fig. S7). The decrease in circularity of the nearest neighbors suggests that weakening the shared junction with the apoptotic cell leads to asymmetry and elongation perpendicular to the radial direction from the defect.

Having identified the characteristics of quiescent, mitotic, and apoptotic cells, and their nearest neighbors, we next compared morphological metrics between cell types before and after these events (Fig. 4F-G, Fig. S8). Prior to mitosis or apoptosis $(t=-30$ minutes $)$, cells generally displayed similar morphology, however, apoptotic cells and nearest neighbors to mitotic cells displayed higher instantaneous speed ( $p=0.0459$ and 0.0379 , respectively), suggesting that changes in cell motility precede these events (Fig. 4F). Interestingly, neighbors of apoptotic cells displayed fewer neighboring cells themselves $(p<0.0001)$, suggesting that apoptosis occurred preferentially in local regions of low cell density. After apoptosis or mitosis ( $t=30$ minutes), daughter cells and nearest neighbors of apoptotic cells displayed distinct morphology compared to quiescent cells (Fig. 4G, S8). Daughter cells remained smaller and displayed lower instantaneous speed ( $p=0.0003$ and 0.0211 , respectively), while nearest 
neighbors of apoptotic cells remained smaller and had fewer cell neighbors $(p=0.0064$ and $<0.0001$, respectively).

\section{iBMEC microvessel response to physical insult by laser ablation}

After defining the hallmarks of iBMEC turnover during homeostasis, we next explored the response of microvessels to physical insult. Various processes can lead to vascular injury, including hemorrhagic stroke (rupture), ischemic stroke (occlusion), neurodegenerative disease (e.g. cerebral amyloid angiopathy), or as a result of treatment (e.g. radiotherapy) [53]. Laser ablation was used to introduce small defects (5 - 10 cells) into iBMEC microvessels, resulting in Evans Blue extravasation at the wound site, similar to the extravasation of blood components observed in vivo following laser ablation of subcortical vessels [54] (Fig. 5A). Similarly sized defects were introduced into 2D monolayers of iBMECs, where the speed of wound healing was very similar between $2 \mathrm{D}$ and 3D $(\tau=\sim 19$ and $\sim 16$ minutes, respectively). In 3D microvessels, complete defect recovery occurred over $\sim 2 \mathrm{~h}$, during which we tracked wound area and the dynamics of individual cells bordering the wound (Fig. 5B-C, Fig. S9). Following closure, cell debris was moved to the center of the defect forming a visible scar that persisted for a further 2 - 4 h (Fig. 5B), however, after 24 h there was no visible evidence of the wound. Since there was no change in the rates of apoptosis, mitosis, and turnover between ablated microvessels and homeostatic controls ( $p=0.414,0.287$, and 0.466 , respectively), repair must occur through a combination of cell migration to the defect and an increase in area of the surrounding cells. Indeed, from cell tracking, we found that cells within about 7 near neighbors of the wound perimeter displayed directed migration towards the centroid of the wound (Fig. S9). This contrasts with apoptotic events where cells beyond the first nearest neighbors display negligible directed motion (Fig. 5D). Additionally, we observed an approximately $15 \%$ increase in average cell area after would closure ( $p=0.0010$, Mann-Whitney test) (Fig. 5E, Fig. S11A-B). As wounds comprised on average $\sim 13 \%$ of the initial imaging frame, this confirms that compensation of lost area occurs predominately via increases in cell size, not proliferation. The number of the smallest cells per unit area (histogram bin: $50-150 \mu \mathrm{m}^{2}$ ) was reduced by more than two-fold following wound closure, suggesting that primarily small cells increase their area to compensate for lost area from the monolayer (Fig. 5E). The cells immediately neighboring the wound displayed no change in area ( $p=0.6095$, Wilcoxon test), but did display decreasing speed ( $p<0.0001$, Wilcoxon test), indicating that repair is mediated at the monolayer level (Fig. 5F, Fig. S10). 

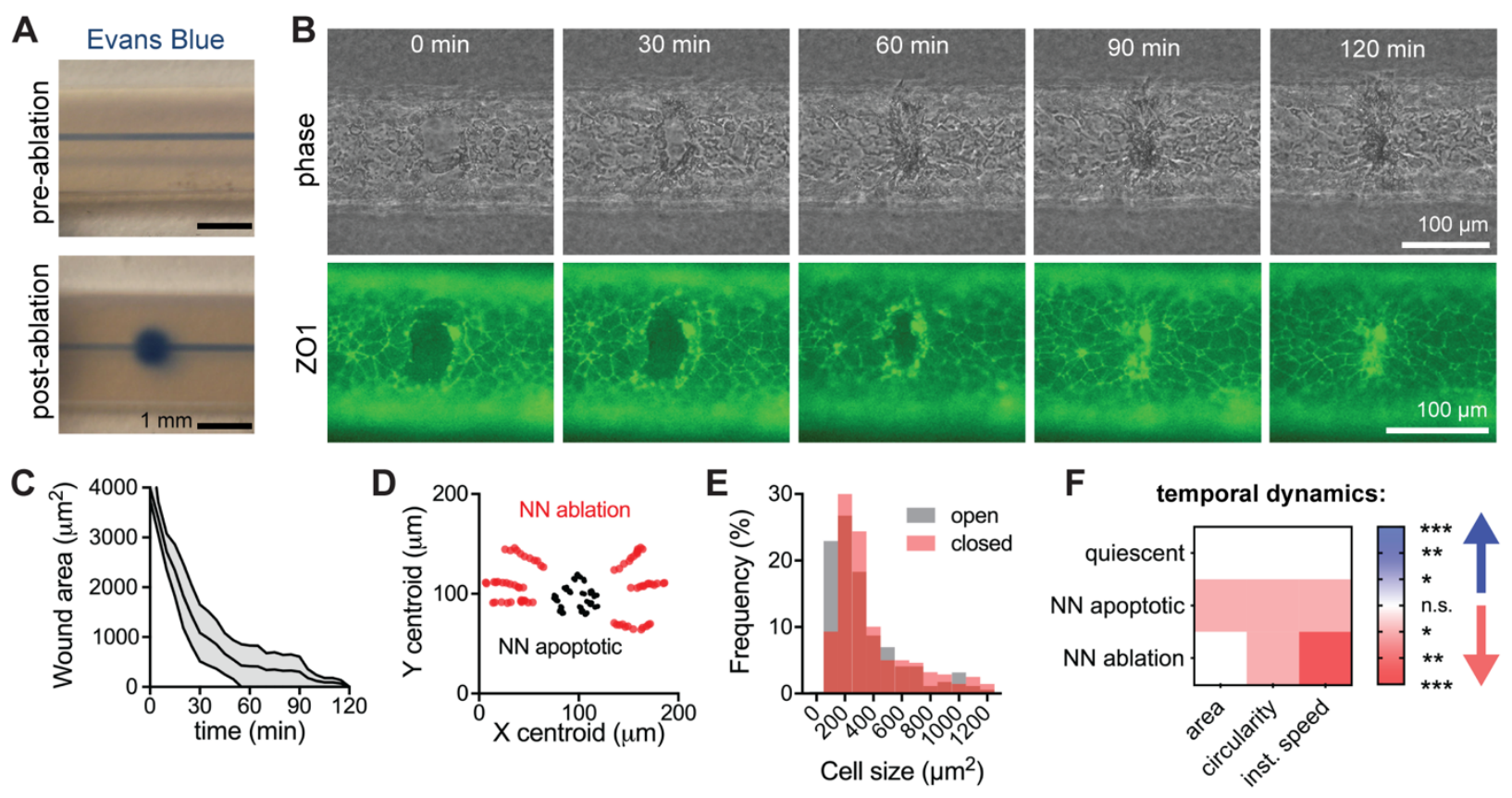

Figure 5. Response of three-dimensional iBMEC microvessels to physical injury.

(A) Representative images of Evans Blue leakage induced by laser ablation of an area corresponding to 5 -10 cells.

(B) Live-cell imaging of microvessel recovery following laser ablation.

(C) Time course of wound size ( $n=4$ microvessels).

(D) Centroid tracking of cell neighbors surrounding a wound during healing (red dots), as well as cell neighbors of an apoptotic cell (black dots). Cell neighbors of ablation display directed migration towards the centroid of the wound.

(E) Cell size distribution immediately after ablation and following wound closure of a total monolayer area of $35,000 \mu \mathrm{m}^{2}(n=353$ cells in open wound and $n=290$ cells in closed wound).

(F) Heatmap comparing changes in cell morphology of nearest neighbors surrounding a wound during healing, relative to quiescent cells and neighbors of an apoptotic cell. Color signifies direction of change, while shade signifies level of significance as calculated using the maximally significant result between a Wilcoxon matched-pairs signed rank test to compare first and last time points and using an $F$ test to determine if linear regression of the morphological time course displayed a statistically non-zero slope. NN - nearest neighbors.

Data are presented as mean \pm SEM. $* p<0.05$, $* * p<0.01$, and $* * * p<0.001$. See also Figure S9-11.

\section{iBMEC microvessel response to chemical injury}

Diverse chemical perturbations are capable of modulating BBB function. To understand mechanisms of chemical injury, we exposed iBMEC microvessels to menadione and melittin. Menadione is a naturally occurring compound that can generate intracellular reactive oxygen species (ROS) and induce oxidative 
stress [55]. In vitro models of oxidative injury to the BBB have revealed increased angiogenic potential, increased solute permeability, and increased rates of cell death in a concentration-dependent manner [56]. Melittin is a membrane active peptide that can increase the permeability of cellular barriers [57, 58]. While both menadione and melittin resulted in rapidly reduced TEER in transwells (data not shown), the mechanisms of barrier loss are not well characterized. Within 3D microvessels, we modeled chronic BBB injury by perfusion with $1 \mathrm{mM}$ menadione and acute BBB injury by 10 -minute exposure to $10 \mu \mathrm{M}$ melittin (Fig. 6A-B). Using simultaneous phase contrast and fluorescence imaging we tracked microvessel structure, barrier properties, and tight junction dynamics.

Both menadione and melittin increased permeability of $10 \mathrm{kDa}$ dextran (Fig. 6A-C) $(p=0.0318$ and 0.0411), but with distinct modes of disruption: menadione induced detachment of the endothelium and partial collapse of the microvessel, whereas melittin induced cell loss from the endothelium (Fig. 6A-B). Endothelial turnover was dramatically increased by melittin $(p=0.0158)$, but not menadione exposure $(p$ $=0.9584)($ Fig. 6D). The increase in turnover in response to melittin was due to an $\sim 3$-fold increase in cell loss $(p=0.0053)$ while the mitosis rate was unchanged $(p=0.3968)$. The cell loss due to melittin exposure resulted in a redistribution of cell area (Fig. 6E, S11C-D); before melittin exposure the cell area was $500 \pm 10 \mu \mathrm{m}^{2}$, while after melittin exposure the cell area increased to $641 \pm 16 \mu \mathrm{m}^{2}(p<0.0001$, Mann-Whitney test). In contrast to apoptotic cells, there was no change in instantaneous speed of lost cells in response to melittin, and no changes in circularity or instantaneous speed for neighbors of lost cells (Fig. 6F). Additionally, we tracked individual cell size and circularity finding that cells lost during melittin exposure had similar area $(p=0.7696)$, but lower circularity $(p<0.0001)$ compared to the entire monolayer (Fig. S11E). The average cell area in the monolayer was also observed to be predictive of melittin response $\left(r^{2}=0.9214\right)$ (Fig. S11F). 
A

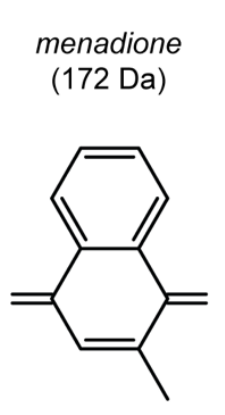

B

melittin

$(2847.5 \mathrm{Da})$

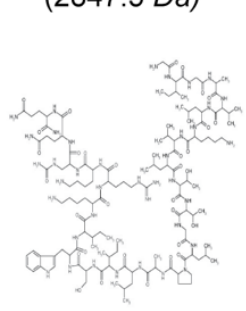

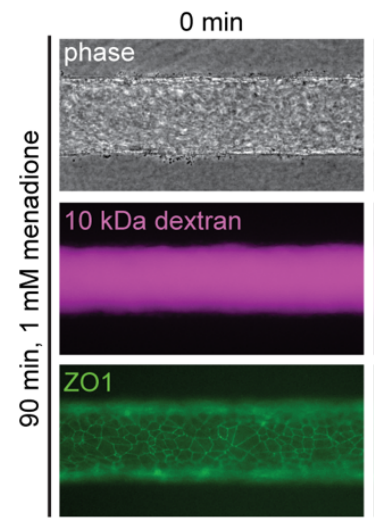

$0 \mathrm{~min}$

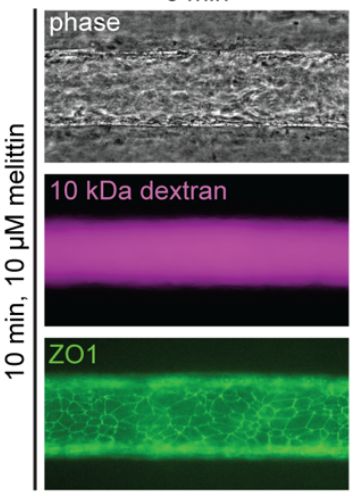

D

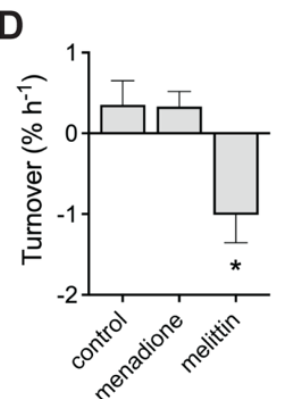

$30 \mathrm{~min}$

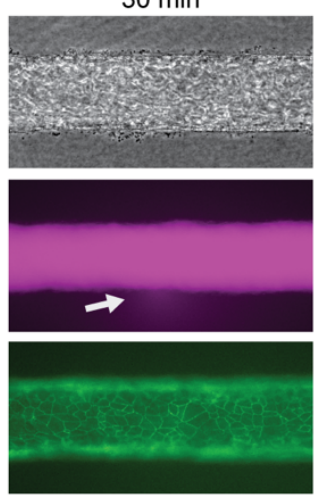

$30 \mathrm{~min}$

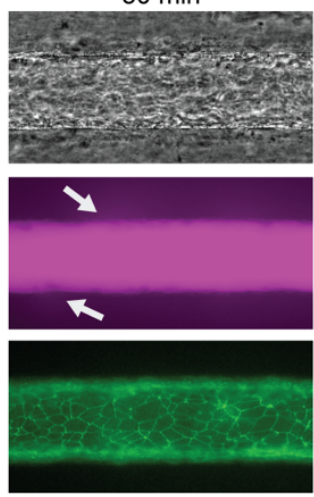

E

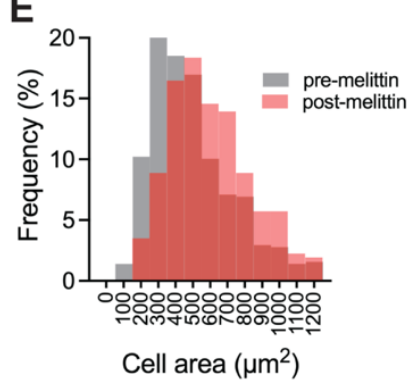

Cell area $\left(\mu \mathrm{m}^{2}\right)$
$60 \mathrm{~min}$

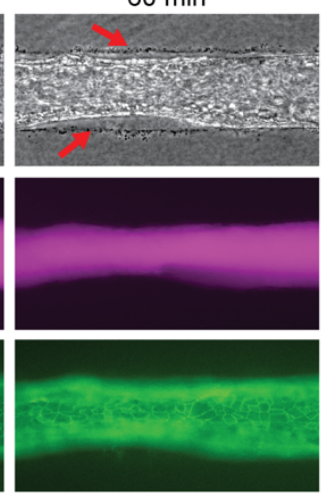

$60 \mathrm{~min}$
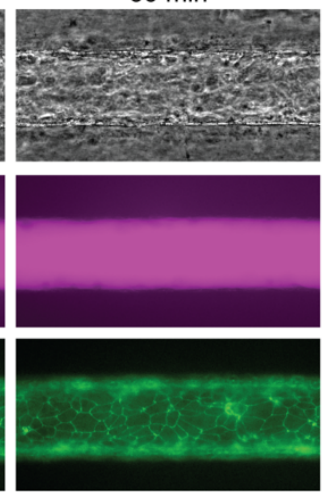

$\mathbf{F}$

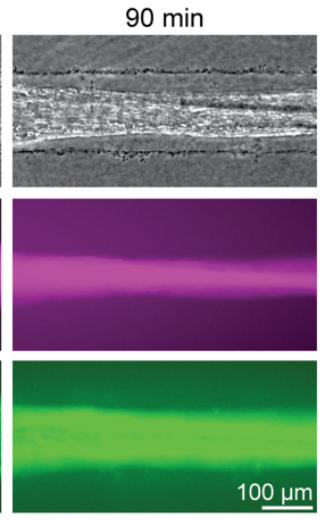

$90 \mathrm{~min}$
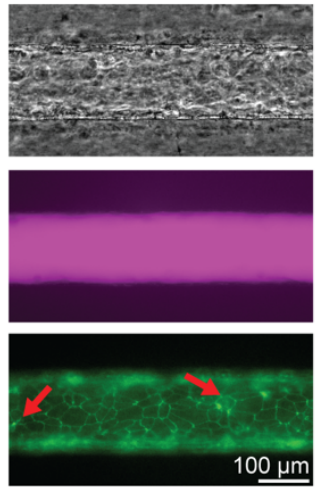

$100 \mu \mathrm{m}$

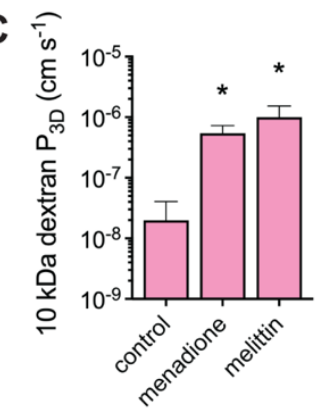$$
\text { 西 }
$$

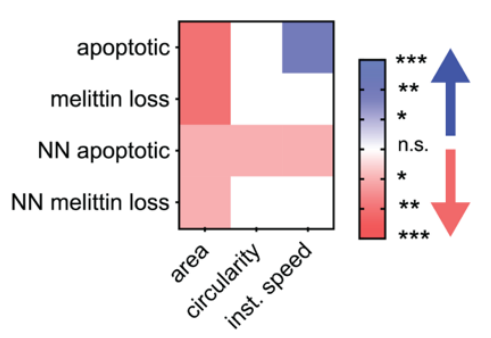

Figure 6. Response of three-dimensional iBMEC microvessels to chemical injury.

(A-B) Real-time imaging of iBMEC microvessels in response to perfusion with menadione or melittin exposure. Red arrows indicate detachment of endothelium (A) and cell collapse (B), respectively, while white arrows denote sites of leakage of $10 \mathrm{kDa}$ dextran (A and B).

(C-D) Comparison of $10 \mathrm{kDa}$ dextran permeability and cell turnover between control $(n=5)$, menadioneexposed $(n=4)$, and melittin-exposed $(n=4)$ iBMEC microvessels. Significance analysis performed using a Kruskal-Wallis test and post hoc Dunn's multiple comparisons test relative to control.

(E) Cell size distribution before and after melittin exposure $(n=581$ cells pre-melittin and 329 cells postmelittin).

(F) Heatmap comparing changes in cell morphology for apoptotic cells and cell loss induced by melittin exposure, as well as their respective nearest neighbors (NN). Color signifies direction of change, while shade signifies level of significance as calculated using the maximally significant result between a 
Wilcoxon matched-pairs signed rank test to compare first and last time points and using an $\mathrm{F}$ test to determine if linear regression of the morphological time course displayed a statistically non-zero slope.

Data are presented as mean \pm SEM. $* p<0.05, * * p<0.01$, and $* * * p<0.001$. See also Figure S10-11.

\section{Discussion}

iBMECs in 2D monolayers recapitulate some aspects of BBB function, however, gene expression profiles show only moderate overlap with human BMECs. Unfortunately, reprogramming iBMECs using ETS transcription factors results in a gene expression profile closer to iECs and dermal ECs, and causes a loss in barrier function [24]. These results highlight the challenge in achieving high transcriptomic similarity to human BMECs while maintaining key phenotypic characteristics of the BBB. However, we show that the 3D microenvironment plays an important role in promoting BBB phenotype, which will extend the repertoire of experiments possible using iBMECs. 3D microvessel models recapitulate direct cell-cell and cell-ECM interactions, shear stress, and cylindrical geometry [26, 59]. Within 3D microvessels, Lucifer yellow permeability was $\sim 2 \times 10^{-7} \mathrm{~cm} \mathrm{~s}^{-1}$, matching permeability values in animal models [60], and approximately 10-fold lower compared to 2D models. Improved barrier function in 3D was not associated with upregulation of tight junctions, suggesting that this effect is mediated by other changes in gene and protein expression. Although endothelial identity was enriched in 3D, expression of many epithelial genes remained unchanged. However, most genes upregulated by the $3 \mathrm{D}$ microenvironment across two independent cell lines were unique from those previously identified to be upregulated by shear stress in 2D monolayers [26]. An important functional implication of these changes is that 3D iBMEC microvessels displayed bFGF-induced angiogenic sprouting and TNF $\alpha$-induced immune cell adhesion, which have been inconsistently reported in 2D iBMEC models.

In previous work we have shown that co-culture with pericytes in $2 \mathrm{D}$ does not increase TEER of iBMEC monolayers that already have high TEER, but can rescue low iBMEC TEER values [61]. Similarly, we have shown that co-culture of pericytes in 3D microvessels does not change solute permeability [61]. However, here we observed that co-culture with pericytes modulated angiogenic and cytokine responses in iBMEC microvessels. Matching in vivo observations [62], we found that pericytes increased the density of angiogenic sprouts from iBMEC microvessels. While in vivo work in pericytedeficient mice finds that pericytes limit leukocyte infiltration in the absence of TNF $\alpha$ exposure [46], here we found that pericytes increase the magnitude of TNF $\alpha$-induced immune cell adhesion, while maintaining low levels of adhesion under baseline conditions. These results suggest that pericytes can 
alter properties of the BBB beyond barrier function, and may guide when pericyte co-culture is critical for in vitro applications.

Real-time imaging of tissue-engineered BBB models enables visualization of a diverse range of processes at the single-cell level. While traditional techniques to probe tight junction dynamics, such as immunocytochemistry, display poor temporal resolution, here we mapped tight junction dynamics during all stages of turnover and in response to physical and chemical insult. Additionally, while 2D assays (e.g. scratch assay) are widely used to study the response of 2D endothelial monolayers to a physical injury, and to infer mechanisms of cell migration and angiogenic potential [63], these models do not enable simultaneous measurements of barrier properties with high spatial resolution.

During homeostasis, cell morphology was stable with small fluctuations in cell-cell junctions. An average of $\sim 6$ nearest neighbors is consistent with the maximum packing of identical disks in 2D (random packing results in five nearest neighbors), where maximizing the number of nearest neighbors may be important in minimizing the probability of paracellular leakage by minimizing the length of cell-cell junctions per unit length of vessel [64]. The processes of apoptosis and mitosis were associated with distinct morphological changes of the cell as well as its neighboring cells, where the underpinnings of these cellular changes were distinct during physical and chemical injury. Notably, the response of neighbors of cells lost from the endothelium was unique (comparison across all conditions summarized in Fig. S10B, C). During apoptosis in homeostatic microvessels, neighboring cells did not directly compensate for the lost area. Instead, neighbors of apoptotic cells displayed decreases in area, circularity, and instantaneous speed during apoptosis. In response to a small wound by laser ablation, repair was associated with directed migration of cells within 7 neighbors to the wound site and the loss of area was compensated by an increase in area of surrounding cells with small initial area. Cells at the perimeter of the wound displayed no change in area but did have dramatic reductions in instantaneous speed as migration decreased. Contrastingly, in response to melittin exposure there was a dramatic increase in cell area across the monolayer which was associated with cell loss and increased paracellular leakage. Cell loss due to melittin was associated with reductions in cell area and the area of neighboring cells, with no other morphological changes.

In summary, through comparison of gene expression and functional properties of iBMECs in 2D monolayers and in tissue-engineered microvessels under a range of conditions, we show how the 3D microenvironment promotes blood-brain barrier phenotype, including enhanced endothelial identity, 
angiogenic response, and cytokine response, which have been inconsistently reported in previous literature. Despite the mixed endothelial and epithelial identity, iBMECs in 3D microenvironments provide a powerful tool for studies of the BBB. Specifically, we applied iBMECs in 3D microvessels to enable detailed visualization and analysis of tight junction dynamics during homeostasis, wound repair, and chemical injury. Our results present new insight into how monolayers of brain microvascular endothelial cells cooperatively respond to mitosis and apoptosis events, as well as to physical or chemical insult.

\section{Methods}

\section{Cell culture and characterization}

BMEC-like cells (iBMECs) were differentiated based on published protocols (see Supplemental Experimental Procedures) [23, 50] from three isogenic WTC iPSC lines (Allen Cell Institute) [65]: enhanced green fluorescence protein (EGFP)-labeled zona occludens-1 (ZO1) (iPSC-TJ), red fluorescence protein (RFP)-labeled plasma membrane (iPSC-PM), and EGFP-labeled $\beta$-actin (iPSC-ACTB). These iPSCs correspond to cell line IDs: AICS-0023 cl.20, AICS-0054 cl.91, and AICS-0016 cl.184, respectively. Additional experiments were conducted on non-isogenic BC1 iBMECs [23, 50]. Isogenic iPSC-derived endothelial cells (iECs) were differentiated using ETV2 modRNA following published protocols [35]. The iECs were cultured in endothelial cell growth medium 2 kit supplemented into basal media (except hydrocortisone, Lonza) with 1x GlutaMax (ThermoFisher) and $10 \mu \mathrm{M}$ SB431542 (Selleckchem). Brain pericyte-like cells (iPCs) were derived through a neural crest intermediate using published protocols (see Supplemental Methods) [36]. The iPCs were cultured in E6 media (StemCell Technologies) supplemented with 10\% FBS and routinely passed using Accutase at 1:5 on tissue-cultured treated surfaces. Primary neonatal human dermal microvascular endothelial cells (HDMECs: Lonza) were cultured in MCDB 131 (Caisson Labs, Carlsbad, CA) supplemented with 10\% heat inactivated fetal bovine serum (Sigma), $25 \mathrm{mg} \mathrm{mL}^{-1}$ endothelial mitogen (Biomedical Technologies), $2 \mathrm{U} \mathrm{mL}^{-1}$ heparin (Sigma), $1 \mu \mathrm{g} \mathrm{mL}^{-1}$ hydrocortisone (Sigma), $0.2 \mathrm{mM}$ ascorbic acid 2-phosphate (49752, Sigma), and 1\% penicillin- streptomycin-glutamine (ThermoFisher). The detailed methods of cell culture, differentiation, barrier assays, and recordings of glucose and oxygen levels during iBMEC differentiation are provided in Supplemental Methods. 


\section{Microvessel fabrication and imaging}

3D microvessels were fabricated similar to previously reported methods [50]. Briefly, $150 \mu \mathrm{m}$ diameter channels were patterned in (1) $7 \mathrm{mg} \mathrm{mL}^{-1}$ collagen I crosslinked with $20 \mathrm{mM}$ genipin, or (2) $6 \mathrm{mg} \mathrm{mL}^{-1}$ collagen I and $1.5 \mathrm{mg} \mathrm{mL}^{-1}$ Matrigel. Channels were connected to inlet and outlet ports within a PDMSbased microfluidic device to control flow rates and shear stress, with perfusion maintained by a gravity driven flow system achieving average flow rates of $\sim 0.25 \mathrm{~mL} \mathrm{~h}^{-1}$. iBMECs (differentiated using $1 \mathrm{~mL}$ media protocols) and iECs were seeded into the channels and allowed to adhere for 30 minutes before initiating perfusion. To form co-cultured microvessels, iPCs were seeded into the channels and perfused at 1 dyne $\mathrm{cm}^{-2}$ for 24 hours prior to seeding iBMECs. 40x confocal images were obtained using a swept field confocal microscope system (Prairie Technologies) with illumination provided by an MLC 400 monolithic laser combiner (Keysight Technologies). 10x epifluorescence images were obtained using an inverted microscope (Nikon Eclipse Ti-E), with illumination was provided by an X-Cite 120LEDBoost (Excelitas Technologies). Time lapse images were acquired every 2 or 5 minutes depending on the experiment, in an environmental chamber maintained at $37{ }^{\circ} \mathrm{C}$ and $5 \% \mathrm{CO}_{2}$.

\section{Quantifying barrier function}

Fluorescence images at the microvessel midplane were acquired every two minutes before (10 minutes total) and after solute perfusion (60 minutes total). Filter cubes (Chroma 39008 and Chroma 41008) were used to independently capture Lucifer yellow (20 ms exposure) and Alexa Fluor-647-conjugated dextran (200 ms exposure), used at concentrations matching 2D assays (see Supplemental Experimental Procedures). Images were collected as ten adjacent frames corresponding to a total image area of 8.18 $\mathrm{mm} \times 0.67 \mathrm{~mm}$. ImageJ was used to plot fluorescence intensity profiles over 70 minutes (36 frames). Permeability $(\mathrm{P})=(\mathrm{r} / 2)(1 / \Delta \mathrm{I})(\mathrm{dI} / \mathrm{dt})_{0}$, where $\mathrm{r}$ is the microvessel radius, $\Delta \mathrm{I}$ is the increase in fluorescence intensity due to luminal filling, and $(\mathrm{dI} / \mathrm{dt})_{0}$ is the rate of fluorescence intensity increase (calculated over 60 minutes) [66]. Images were segmented into ten adjacent regions-of-interest (ROIs), where the permeability is reported as the mean value of the five adjacent frames surrounding the minimum to minimize artifacts from interstitial dye entering the matrix from inlet and outlet ports during imaging.

\section{Bulk RNA sequencing}


RNA was collected two days after subculture or passing. Cells were washed with PBS and then lysed using RLT buffer supplemented with 1\% $\beta$-mercaptoethanol. Lysates were eluted with RNase-free water after purification using a RNeasy Mini Kit (Qiagen) and DNase I digestion, following manufacturer instructions. All samples had an RNA integrity number $>8.4$ as measured by an Agilent 2100 bioanalyzer. Total RNA was subjected to oligo (dT) capture and enrichment, and the resulting mRNA fraction was used to construct cDNA libraries (performed by Novogene). Sequencing was carried out on an Illumina NovoSeq platform (performed by Novogene) with paired end $150 \mathrm{bp}$ reads, generating approximately 20 million paired reads per sample. The R (v4.0.1) package Rsubread (v2.0.1) was used for raw read alignment and for read quantification to the reference human genome (GRCh38) [67]. The R package DESeq2 (v1.28.1) was used for normalization, visualization, and differential analysis [68]. Raw reads were normalized using the DESeq2 variance stabilizing regularized logarithm (rlog) transformation prior to calculation of Euclidean sample distances, Spearman correlation, and principal component analysis (PCA). Differentially expressed genes (DEGs) were determined using the Wald test with BenjaminiHochberg correction, where adjusted $p$ values $<0.05$ was considered statistically significant. Pathway enrichment analysis (GO biological process terms) was conducted by inputting DEGs with $>1 \log _{2} \mathrm{FC}$ enrichment into Enrichr [69]. Correlation, PCA, volcano, and lollipop plots were all formatted using the R packages ggplot2 (v3.3.2), ggrepel (v0.8.2), pheatmap (v1.0.12), and RColorBrewer (v1.1-2). Data are deposited at NCBI, and published bulk RNA-seq data (FASTQ files; see Table S1) were obtained from the Gene Expression Omnibus (GEO).

\section{Immunocytochemistry}

For 2D experiments, iBMECs were seeded at 250,000 cells $\mathrm{cm}^{-2}$ on borosilicate cover glass slides (Thermo Scientific); for 3D experiments, iBMECs were seeded as described above. Two days later, cells were washed with phosphate-buffered saline (PBS; ThermoFisher), fixed with ice-cold methanol for 15 minutes, and blocked with 10\% goat serum (Cell Signaling Technology) or 10\% donkey serum (Millipore Sigma), supplemented with 0.1\% Triton X-100 (Millipore Sigma) for 30 minutes. Primary antibodies and dilutions are listed in Table S2. After three washes with PBS, cells were treated with Alexa Flour-647 and Alexa Flour-488 secondary antibodies (Life Technologies) diluted 1:200 in blocking buffer for 1 hour at room temperature. Nuclei were stained using $1 \mu \mathrm{g} \mathrm{mL} \mathrm{m}^{-1}$ DAPI (ThermoFisher). Confocal images were acquired at 40x magnification as previously reported [50]. Control images were also collected without primary antibody to confirm fluorescence above non-specific background. Semi-quantitative analysis of 
protein levels between 2D and 3D was calculated based on the ratio of immunofluorescence between 3D and 2D, and normalized to the ratio of nucleus fluorescence (by DAPI staining) between 3D and 2D, and normalized by surface area of endothelial surface area.

\section{Quantifying angiogenic and cytokine response}

To probe angiogenic response, microvessels with and without pericytes were formed on $6 \mathrm{mg} \mathrm{mL}^{-1}$ collagen I and $1.5 \mathrm{mg} \mathrm{mL}^{-1}$ Matrigel hydrogels. Genipin crosslinking was not used for these studies as the increased stiffness suppresses angiogenic sprouting. As a static control, we lined microspheres with iBMECs and embedded them within hydrogels as previous reported [48]. After $48 \mathrm{~h}$ treatment with 20 ng $\mathrm{mL}^{-1}$ bFGF (R\&D Systems), sprouts were manually counted in ImageJ, where "sprout length" represents the total length of sprouts per unit area of endothelium.

To probe cytokine responses, microvessels with and without pericytes were formed in $7 \mathrm{mg} \mathrm{mL}^{-1}$ collagen I crosslinked with 20 mM genipin. THP-1 (ATCC® TIB-202 ${ }^{\mathrm{TM}}$ ), a human leukemia monocytic cell line [70], were grown in suspension with RPMI-1640 Medium (Sigma) supplemented with 10\% fetal bovine serum (Sigma) and 1\% penicillin-streptomycin. Cells were labeled with $1 \mu \mathrm{M}$ CellTracker ${ }^{\mathrm{TM}} \operatorname{Red}$ CMTPX Dye (ThermoFisher) in serum-free media for $20 \mathrm{~min}$, and then resuspended at $1 \times 10^{6}$ cells $\mathrm{mL}^{-1}$ in complete media. Microvessels with and without $24 \mathrm{~h}$ treatment with $5 \mathrm{ng} \mathrm{mL}{ }^{-1} \mathrm{TNF} \alpha$, were perfused

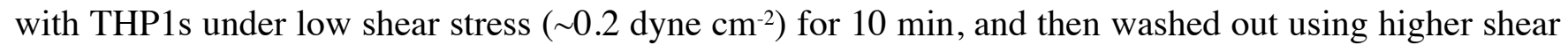
stress $\left(\sim 2\right.$ dyne $\left.\mathrm{cm}^{-2}\right)$. As a 2D control, we exposed monolayers of iBMECs to THP1s matching microvessel conditions (except static culture) and mimicked washout by conducting three media switches. Adherent immune cells were manually counted in ImageJ and normalized to unit area of endothelium. All TNF $\alpha$ experiments were conducted on iBMECs differentiated from BC1 iPSCs. Bulk RNA was extracted from microvessels $24 \mathrm{~h}$ after $5 \mathrm{ng} \mathrm{mL} \mathrm{m}^{-1} \mathrm{TNF} \alpha$ exposure.

\section{Quantifying tight junction dynamics}

Images were collected every 5 minutes of Hoescht-33342 (ThermoFisher)-labeled nuclei, EGFP-labeled ZO1, and phase contrast. Endothelial cell dynamics within the monolayer were quantified using previously developed tools in Image $(\mathrm{NIH})$ [50, 71]. From a total of 1871 cells, all apoptotic and mitotic cells with 30 minutes of imaging before and after these events were included in analysis; control cells 
were selected randomly. Rates of apoptosis, mitosis, and turnover are not statistically significantly different between measurements based on phase contrast (as previously utilized [43]) or ZO1 fluorescence ( $p>0.05$ for all comparisons). Using ZO1 traces, cell area $\left(\mu \mathrm{m}^{2}\right)$, perimeter $(\mu \mathrm{m})$, circularity, aspect ratio, and centroid location of individual cells were then calculated from freehand tracings of cell boundaries outlined by tight junctions visible under fluorescence. The number of nearest cell neighbors were manually counted based on based edges of tight junction expression. Instantaneous cell speed (nm s${ }^{-1}$ ) was calculated from the change in the location of the centroid of the cell divided by the elapsed time. The cell speed in the $\mathrm{x}$-direction (along the length of the vessel and in the direction of flow) was determined from the total change in position in the $\mathrm{x}$-direction divided by the elapsed time. Results were validated across independent researchers to confirm robustness of technique (Fig. S6D). PCA of all morphological metrics was conducted using the prcomp function in the $\mathrm{R}$ stats package (v 3.6.2). Metrics were first shifted to be zero-centered and scaled to have unit variance. Contributions of morphological metrics to principal components $1-4$ were quantified using the squared cosine metric (Fig. S6C).

\section{Modeling physical and chemical injury}

Laser ablation was conducted via $90 \mathrm{~s}$ irradiation at $2100 \mathrm{~mW}$ using the $750 \mathrm{~nm}$ line of an LSM 510 laser scanning microscope (Zeiss), where a rectangular prism (50 $\mu \mathrm{m}$ width x $150 \mu \mathrm{m}$ length x $20 \mu \mathrm{m}$ height) at the bottom plane of microvessels was irradiated. For 2D studies, iBMEC monolayers were scratched with a pipette tip to achieve a similar defect area. The defect area $\left(\mu \mathrm{m}^{2}\right)$ was determined by tracing the perimeter of the wound at each time point. Barrier function was also assessed following perfusion with $0.0683 \% \mathrm{w} / \mathrm{v}$ Evans blue (in medium), matching concentrations in the bloodstream of adult mice dosed at $1 \%$ weight/volume [72]. To model chemical injury, microvessels were perfused with $1 \mathrm{mM}$ menadione (Sigma) for 90 minutes or $10 \mu \mathrm{M}$ melittin with a free carboxy C-terminus (melittin-COO-) (Bio-Synthesis Inc) for 10 minutes. Simultaneously, microvessels were perfused with $10 \mathrm{kDa}$ dextran with phase and fluorescence imaging conducted over 90 minutes.

\section{Statistical analysis}

Statistical testing was performed using Prism ver. 8 (GraphPad). All experimental values are reported as mean \pm standard error of the mean (SEM). Differentiations in which $1 \mathrm{~mL}$ volume produced peak TEER below $1500 \Omega \mathrm{cm}^{2}$ ( 10\% frequency) were excluded from analysis. Statistical tests were chosen based 
on paired status and normality (Shapiro-Wilk test), where details are provided in figure captions. Differences were considered statistically significant with the following thresholds: $* \mathrm{p}<0.05, * * \mathrm{p}<0.01$, $* * * \mathrm{p}<0.001$, and $* * * * \mathrm{p}<0.0001$.

\section{Data and materials availability}

All data associated with this study are available in the main text or the supplementary materials. The raw data required to reproduce these findings are available from the corresponding author. RNA sequencing data are deposited in GEO.

\section{Acknowledgements}

The authors also acknowledge the assistance of Alanna Farrell and Erin Pryce. Funding: This work was supported by DTRA (HDTRA1-15-1-0046) and NIH (R01NS106008). RML acknowledges a National Science Foundation Graduate Research Fellowship under Grant No. DGE1746891 and the support of Jeffrey Herman during long breaks from the benchtop during the COVID-19 pandemic.

\section{Author contributions}

RML: Conceptualization, Investigation, Formal analysis, Writing - Original Draft, Writing - Review \& Editing, Supervision; MS, GNG, RFN, RY, JGD, JZ, RJ, JJJ, NZ: Investigation; PCS: Conceptualization, Writing - Review \& Editing, Supervision, Funding acquisition

\section{Competing interests.}

The authors declare no competing interests. 


\section{References}

[1] J.G. DeStefano, J.J. Jamieson, R.M. Linville, P.C. Searson, Benchmarking in vitro tissue-engineered blood-brain barrier models, Fluids Barriers CNS 15(1) (2018) 32.

[2] W.M. Pardridge, The blood-brain barrier: bottleneck in brain drug development, NeuroRx 2(1) (2005) 3-14.

[3] D.S. Hersh, A.S. Wadajkar, N. Roberts, J.G. Perez, N.P. Connolly, V. Frenkel, J.A. Winkles, G.F. Woodworth, A.J. Kim, Evolving Drug Delivery Strategies to Overcome the Blood Brain Barrier, Curr Pharm Des 22(9) (2016) 1177-1193.

[4] C. Greene, J. Kealy, M.M. Humphries, Y. Gong, J. Hou, N. Hudson, L.M. Cassidy, R. Martiniano, V. Shashi, S.R. Hooper, G.A. Grant, P.F. Kenna, K. Norris, C.K. Callaghan, M.D. Islam, S.M. O'Mara, Z. Najda, S.G. Campbell, J.S. Pachter, J. Thomas, N.M. Williams, P. Humphries, K.C. Murphy, M. Campbell, Dose-dependent expression of claudin-5 is a modifying factor in schizophrenia, Mol Psychiatry 23(11) (2018) 2156-2166.

[5] H.W. Song, K.L. Foreman, B.D. Gastfriend, J.S. Kuo, S.P. Palecek, E.V. Shusta, Transcriptomic comparison of human and mouse brain microvessels, Sci Rep 10(1) (2020) 12358.

[6] N.M. O'Brown, S.J. Pfau, C. Gu, Bridging barriers: a comparative look at the blood-brain barrier across organisms, Genes Dev 32(7-8) (2018) 466-478.

[7] E. Neuwelt, N. Abbott, L. Abrey, W.A. Banks, B. Blakley, T. Davis, B. Engelhardt, P. Grammas, M. Nedergaard, J. Nutt, W. Pardridge, G.A. Rosenberg, Q. Smith, L.R. Drewes, Strategies to advance translational research into brain barriers, Lancet Neurol 7(1) (2008) 84-96.

[8] E.A. Neuwelt, B. Bauer, C. Fahlke, G. Fricker, C. Iadecola, D. Janigro, L. Leybaert, Z. Molnar, M.E. O'Donnell, J.T. Povlishock, N.R. Saunders, F. Sharp, D. Stanimirovic, R.J. Watts, L.R. Drewes, Engaging neuroscience to advance translational research in brain barrier biology, Nature Reviews Neuroscience 12(3) (2011) 169-82.

[9] M.J. Ochocinska, B.V. Zlokovic, P.C. Searson, A.T. Crowder, R.P. Kraig, J.Y. Ljubimova, T.G. Mainprize, W.A. Banks, W. Timmer, R.Q. Warren, A. Kindzelski, C.H. Liu, NIH Workshop Report on the Trans-Agency Blood-Brain Interface Workshop 2016, Fluids \& Barriers of the CNS 14 (2017) 12.

[10] A. Herland, A.D. van der Meer, E.A. FitzGerald, T.E. Park, J.J. Sleeboom, D.E. Ingber, Distinct Contributions of Astrocytes and Pericytes to Neuroinflammation Identified in a 3D Human Blood-Brain Barrier on a Chip, PLoS One 11(3) (2016) e0150360.

[11] Q.R. Smith, S.I. Rapoport, Cerebrovascular permeability coefficients to sodium, potassium, and chloride, J Neurochem 46(6) (1986) 1732-42.

[12] C. Crone, S.P. Olesen, Electrical resistance of brain microvascular endothelium, Brain Res 241(1) (1982) 49-55.

[13] D.E. Eigenmann, G. Xue, K.S. Kim, A.V. Moses, M. Hamburger, M. Oufir, Comparative study of four immortalized human brain capillary endothelial cell lines, hCMEC/D3, hBMEC, TY10, and BB19, and optimization of culture conditions, for an in vitro blood-brain barrier model for drug permeability studies, Fluids Barriers CNS 10(1) (2013) 33.

[14] B.P. Daniels, L. Cruz-Orengo, T.J. Pasieka, P.O. Couraud, I.A. Romero, B. Weksler, J.A. Cooper, T.L. Doering, R.S. Klein, Immortalized human cerebral microvascular endothelial cells maintain the properties of primary cells in an in vitro model of immune migration across the blood brain barrier, $\mathrm{J}$ Neurosci Meth 212(1) (2013) 173-179.

[15] N.A. Khan, Novel in vitro and in vivo models to study central nervous system infections due to Acanthamoeba spp., Exp Parasitol 126(1) (2010) 69-72. 
[16] A. Reichel, D.J. Begley, N.J. Abbottt, An overview of in vitro techniques for blood brain barrier studies, in: S. Nag (Ed.), Methods in Molecular Medicine, V. 89: The Blood Brain Barrier, Humana, Totowa, NJ, 2003, pp. 307-324.

[17] M.F. Sabbagh, J. Nathans, A genome-wide view of the de-differentiation of central nervous system endothelial cells in culture, eLife 9 (2020).

[18] M.J. Workman, C.N. Svendsen, Recent advances in human iPSC-derived models of the blood-brain barrier, Fluids Barriers CNS 17(1) (2020) 30.

[19] E.S. Lippmann, S.M. Azarin, J.E. Kay, R.A. Nessler, H.K. Wilson, A. Al-Ahmad, S.P. Palecek, E.V. Shusta, Derivation of blood-brain barrier endothelial cells from human pluripotent stem cells, Nat Biotechnol 30(8) (2012) 783-91.

[20] E.S. Lippmann, A. Al-Ahmad, S.M. Azarin, S.P. Palecek, E.V. Shusta, A retinoic acid-enhanced, multicellular human blood-brain barrier model derived from stem cell sources, Sci Rep 4 (2014) 4160.

[21] M.J. Stebbins, E.S. Lippmann, M.G. Faubion, R. Daneman, S.P. Palecek, E.V. Shusta, Activation of RARalpha, RARgamma, or RXRalpha Increases Barrier Tightness in Human Induced Pluripotent Stem Cell-Derived Brain Endothelial Cells, Biotechnol J 13(2) (2018).

[22] T. Qian, S.E. Maguire, S.G. Canfield, X. Bao, W.R. Olson, E.V. Shusta, S.P. Palecek, Directed differentiation of human pluripotent stem cells to blood-brain barrier endothelial cells, Sci Adv 3(11) (2017) e1701679.

[23] M.E. Katt, Z.S. Xu, S. Gerecht, P.C. Searson, Human Brain Microvascular Endothelial Cells Derived from the BC1 iPS Cell Line Exhibit a Blood-Brain Barrier Phenotype, PLoS One 11(4) (2016) e0152105. [24] T.M. Lu, S. Houghton, T. Magdeldin, J.G.B. Duran, A.P. Minotti, A. Snead, A. Sproul, D.T. Nguyen, J. Xiang, H.A. Fine, Z. Rosenwaks, L. Studer, S. Rafii, D. Agalliu, D. Redmond, R. Lis, Pluripotent stem cell-derived epithelium misidentified as brain microvascular endothelium requires ETS factors to acquire vascular fate, Proc Natl Acad Sci U S A 118(8) (2021).

[25] F. Roudnicky, B.K. Kim, Y. Lan, R. Schmucki, V. Kuppers, K. Christensen, M. Graf, C. Patsch, M. Burcin, C.A. Meyer, P.D. Westenskow, C.A. Cowan, Identification of a combination of transcription factors that synergistically increases endothelial cell barrier resistance, Sci Rep 10(1) (2020) 3886.

[26] G.D. Vatine, R. Barrile, M.J. Workman, S. Sances, B.K. Barriga, M. Rahnama, S. Barthakur, M. Kasendra, C. Lucchesi, J. Kerns, N. Wen, W.R. Spivia, Z. Chen, J. Van Eyk, C.N. Svendsen, Human iPSC-Derived Blood-Brain Barrier Chips Enable Disease Modeling and Personalized Medicine Applications, Cell Stem Cell 24(6) (2019) 995-1005 e6.

[27] T.E. Park, N. Mustafaoglu, A. Herland, R. Hasselkus, R. Mannix, E.A. FitzGerald, R. Prantil-Baun, A. Watters, O. Henry, M. Benz, H. Sanchez, H.J. McCrea, L.C. Goumnerova, H.W. Song, S.P. Palecek, E. Shusta, D.E. Ingber, Hypoxia-enhanced Blood-Brain Barrier Chip recapitulates human barrier function and shuttling of drugs and antibodies, Nat Commun 10(1) (2019) 2621.

[28] G.N. Grifno, A.M. Farrell, R.M. Linville, D. Arevalo, J.H. Kim, L. Gu, P.C. Searson, Tissueengineered blood-brain barrier models via directed differentiation of human induced pluripotent stem cells, Sci Rep 9(1) (2019) 13957.

[29] E.K. Hollmann, A.K. Bailey, A.V. Potharazu, M.D. Neely, A.B. Bowman, E.S. Lippmann, Accelerated differentiation of human induced pluripotent stem cells to blood-brain barrier endothelial cells, Fluids Barriers CNS 14(1) (2017) 9.

[30] E.H. Neal, N.A. Marinelli, Y. Shi, P.M. McClatchey, K.M. Balotin, D.R. Gullett, K.A. Hagerla, A.B. Bowman, K.C. Ess, J.P. Wikswo, E.S. Lippmann, A Simplified, Fully Defined Differentiation Scheme for Producing Blood-Brain Barrier Endothelial Cells from Human iPSCs, Stem Cell Reports 12(6) (2019) 1380-1388. 
[31] P. Motallebnejad, S.M. Azarin, Chemically defined human vascular laminins for biologically relevant culture of hiPSC-derived brain microvascular endothelial cells, Fluids Barriers CNS 17(1) (2020) 54.

[32] H. Aoki, M. Yamashita, T. Hashita, T. Iwao, T. Matsunaga, Laminin 221 fragment is suitable for the differentiation of human induced pluripotent stem cells into brain microvascular endothelial-like cells with robust barrier integrity, Fluids Barriers CNS 17(1) (2020) 25.

[33] R. Patel, A.J. Alahmad, Growth-factor reduced Matrigel source influences stem cell derived brain microvascular endothelial cell barrier properties, Fluids Barriers CNS 13 (2016) 6.

[34] H.K. Wilson, S.G. Canfield, M.K. Hjortness, S.P. Palecek, E.V. Shusta, Exploring the effects of cell seeding density on the differentiation of human pluripotent stem cells to brain microvascular endothelial cells, Fluids Barriers CNS 12 (2015) 13.

[35] K. Wang, R.Z. Lin, X. Hong, A.H. Ng, C.N. Lee, J. Neumeyer, G. Wang, X. Wang, M. Ma, W.T. $\mathrm{Pu}$, G.M. Church, J.M. Melero-Martin, Robust differentiation of human pluripotent stem cells into endothelial cells via temporal modulation of ETV2 with modified mRNA, Sci Adv 6(30) (2020) eaba7606. [36] M.J. Stebbins, B.D. Gastfriend, S.G. Canfield, M.S. Lee, D. Richards, M.G. Faubion, W.J. Li, R. Daneman, S.P. Palecek, E.V. Shusta, Human pluripotent stem cell-derived brain pericyte-like cells induce blood-brain barrier properties, Sci Adv 5(3) (2019) eaau7375.

[37] Y. Zhang, S.A. Sloan, L.E. Clarke, C. Caneda, C.A. Plaza, P.D. Blumenthal, H. Vogel, G.K. Steinberg, M.S. Edwards, G. Li, J.A. Duncan, 3rd, S.H. Cheshier, L.M. Shuer, E.F. Chang, G.A. Grant, M.G. Gephart, B.A. Barres, Purification and Characterization of Progenitor and Mature Human Astrocytes Reveals Transcriptional and Functional Differences with Mouse, Neuron 89(1) (2016) 37-53.

[38] F. Roudnicky, J.D. Zhang, B.K. Kim, N.J. Pandya, Y. Lan, L. Sach-Peltason, H. Ragelle, P. Strassburger, S. Gruener, M. Lazendic, S. Uhles, F. Revelant, O. Eidam, G. Sturm, V. Kueppers, K. Christensen, L.D. Goldstein, M. Tzouros, B. Banfai, Z. Modrusan, M. Graf, C. Patsch, M. Burcin, C.A. Meyer, P.D. Westenskow, C.A. Cowan, Inducers of the endothelial cell barrier identified through chemogenomic screening in genome-edited hPSC-endothelial cells, Proc Natl Acad Sci U S A 117(33) (2020) 19854-19865.

[39] K.R. Kalari, K.J. Thompson, A.A. Nair, X. Tang, M.A. Bockol, N. Jhawar, S.K. Swaminathan, V.J. Lowe, K.K. Kandimalla, BBBomics-Human Blood Brain Barrier Transcriptomics Hub, Front Neurosci 10 (2016) 71.

[40] C. Ghosh, J. Gonzalez-Martinez, M. Hossain, L. Cucullo, V. Fazio, D. Janigro, N. Marchi, Pattern of P450 expression at the human blood-brain barrier: roles of epileptic condition and laminar flow, Epilepsia 51(8) (2010) 1408-17.

[41] R. Magid, T.J. Murphy, Z.S. Galis, Expression of matrix metalloproteinase-9 in endothelial cells is differentially regulated by shear stress. Role of c-Myc, J Biol Chem 278(35) (2003) 32994-9.

[42] D.E. Conway, S. Lee, S.G. Eskin, A.K. Shah, H. Jo, L.V. McIntire, Endothelial metallothionein expression and intracellular free zinc levels are regulated by shear stress, Am J Physiol Cell Physiol 299(6) (2010) C1461-7.

[43] J.G. DeStefano, Z.S. Xu, A.J. Williams, N. Yimam, P.C. Searson, Effect of shear stress on iPSCderived human brain microvascular endothelial cells (dhBMECs), Fluids Barriers CNS 14(1) (2017) 20.

[44] F.J. Garcia, N. Sun, H. Lee, B. Godlewski, K. Galani, J. Mantero, D.A. Bennett, M. Sahin, M. Kellis, M. Heiman, Single-cell dissection of the human cerebrovasculature in health and disease, bioRxiv (2021) 2021.04.26.440975.

[45] F. Girolamo, I. de Trizio, M. Errede, G. Longo, A. d'Amati, D. Virgintino, Neural crest cell-derived pericytes act as pro-angiogenic cells in human neocortex development and gliomas, Fluids Barriers CNS 18(1) (2021) 14. 
[46] O. Torok, B. Schreiner, J. Schaffenrath, H.C. Tsai, U. Maheshwari, S.A. Stifter, C. Welsh, A. Amorim, S. Sridhar, S.G. Utz, W. Mildenberger, S. Nassiri, M. Delorenzi, A. Aguzzi, M.H. Han, M. Greter, B. Becher, A. Keller, Pericytes regulate vascular immune homeostasis in the CNS, Proc Natl Acad Sci U S A 118(10) (2021).

[47] T.Y. Kang, F. Bocci, M.K. Jolly, H. Levine, J.N. Onuchic, A. Levchenko, Pericytes enable effective angiogenesis in the presence of proinflammatory signals, Proc Natl Acad Sci U S A 116(47) (2019) 2355123561.

[48] R.M. Linville, D. Arevalo, J.C. Maressa, N. Zhao, P.C. Searson, Three-dimensional induced pluripotent stem-cell models of human brain angiogenesis, Microvasc Res 132 (2020) 104042.

[49] H. Nishihara, B.D. Gastfriend, S. Soldati, S. Perriot, A. Mathias, Y. Sano, F. Shimizu, F. Gosselet, T. Kanda, S.P. Palecek, R. Du Pasquier, E.V. Shusta, B. Engelhardt, Advancing human induced pluripotent stem cell-derived blood-brain barrier models for studying immune cell interactions, FASEB J 34(12) (2020) 16693-16715.

[50] R.M. Linville, J.G. DeStefano, M.B. Sklar, Z. Xu, A.M. Farrell, M.I. Bogorad, C. Chu, P. Walczak, L. Cheng, V. Mahairaki, K.A. Whartenby, P.A. Calabresi, P.C. Searson, Human iPSC-derived blood-brain barrier microvessels: validation of barrier function and endothelial cell behavior, Biomaterials 190-191 (2019) 24-37.

[51] C.M. Van Itallie, A.S. Fanning, A. Bridges, J.M. Anderson, ZO-1 stabilizes the tight junction solute barrier through coupling to the perijunctional cytoskeleton, Mol Biol Cell 20(17) (2009) 3930-40.

[52] J. Rosenblatt, M.C. Raff, L.P. Cramer, An epithelial cell destined for apoptosis signals its neighbors to extrude it by an actin- and myosin-dependent mechanism, Curr Biol 11(23) (2001) 1847-57.

[53] M.I. Bogorad, J.G. DeStefano, R.M. Linville, A.D. Wong, P.C. Searson, Cerebrovascular plasticity: Processes that lead to changes in the architecture of brain microvessels, J Cereb Blood Flow Metab 39(8) (2019) 1413-1432.

[54] N. Nishimura, C.B. Schaffer, B. Friedman, P.S. Tsai, P.D. Lyden, D. Kleinfeld, Targeted insult to subsurface cortical blood vessels using ultrashort laser pulses: three models of stroke, Nat Methods 3(2) (2006) 99-108.

[55] G. Loor, J. Kondapalli, J.M. Schriewer, N.S. Chandel, T.L. Vanden Hoek, P.T. Schumacker, Menadione triggers cell death through ROS-dependent mechanisms involving PARP activation without requiring apoptosis, Free Radic Biol Med 49(12) (2010) 1925-36.

[56] C. Anasooya Shaji, B.D. Robinson, A. Yeager, M.R. Beeram, M.L. Davis, C.L. Isbell, J.H. Huang, B. Tharakan, The Tri-phasic Role of Hydrogen Peroxide in Blood-Brain Barrier Endothelial cells, Sci Rep 9(1) (2019) 133.

[57] G. Wiedman, K. Herman, P. Searson, W.C. Wimley, K. Hristova, The electrical response of bilayers to the bee venom toxin melittin: evidence for transient bilayer permeabilization, Biochim Biophys Acta 1828(5) (2013) 1357-64.

[58] R.M. Linville, A. Komin, X. Lan, J.G. DeStefano, C. Chu, G. Liu, P. Walczak, K. Hristova, P.C. Searson, Reversible blood-brain barrier opening utilizing the membrane active peptide melittin in vitro and in vivo, Biomaterials 275 (2021) 120942.

[59] S.G. Canfield, M.J. Stebbins, M.G. Faubion, B.D. Gastfriend, S.P. Palecek, E.V. Shusta, An isogenic neurovascular unit model comprised of human induced pluripotent stem cell-derived brain microvascular endothelial cells, pericytes, astrocytes, and neurons, Fluids Barriers CNS 16(1) (2019) 25.

[60] A.S. Easton, M.H. Sarker, P.A. Fraser, Two components of blood-brain barrier disruption in the rat, J Physiol 503 ( Pt 3) (1997) 613-23. 
[61] J.J. Jamieson, R.M. Linville, Y.Y. Ding, S. Gerecht, P.C. Searson, Role of iPSC-derived pericytes on barrier function of iPSC-derived brain microvascular endothelial cells in 2D and 3D, Fluids Barriers CNS 16(1) (2019) 15.

[62] H.M. Eilken, R. Dieguez-Hurtado, I. Schmidt, M. Nakayama, H.W. Jeong, H. Arf, S. Adams, N. Ferrara, R.H. Adams, Pericytes regulate VEGF-induced endothelial sprouting through VEGFR1, Nat Commun 8(1) (2017) 1574.

[63] L.G. Rodriguez, X. Wu, J.L. Guan, Wound-healing assay, Methods Mol Biol 294 (2005) 23-9.

[64] M. Ye, H.M. Sanchez, M. Hultz, Z. Yang, M. Bogorad, A.D. Wong, P.C. Searson, Brain microvascular endothelial cells resist elongation due to curvature and shear stress, Sci Rep 4 (2014) 4681. [65] F.R. Kreitzer, N. Salomonis, A. Sheehan, M. Huang, J.S. Park, M.J. Spindler, P. Lizarraga, W.A. Weiss, P.L. So, B.R. Conklin, A robust method to derive functional neural crest cells from human pluripotent stem cells, Am J Stem Cells 2(2) (2013) 119-31.

[66] V.H. Huxley, F.E. Curry, R.H. Adamson, Quantitative fluorescence microscopy on single capillaries: alpha-lactalbumin transport, Am J Physiol 252(1 Pt 2) (1987) H188-97.

[67] Y. Liao, G.K. Smyth, W. Shi, The R package Rsubread is easier, faster, cheaper and better for alignment and quantification of RNA sequencing reads, Nucleic Acids Res 47(8) (2019) e47.

[68] M.I. Love, W. Huber, S. Anders, Moderated estimation of fold change and dispersion for RNA-seq data with DESeq2, Genome Biol 15(12) (2014) 550.

[69] E.Y. Chen, C.M. Tan, Y. Kou, Q. Duan, Z. Wang, G.V. Meirelles, N.R. Clark, A. Ma'ayan, Enrichr: interactive and collaborative HTML5 gene list enrichment analysis tool, BMC Bioinformatics 14 (2013) 128.

[70] W. Chanput, J.J. Mes, H.J. Wichers, THP-1 cell line: an in vitro cell model for immune modulation approach, Int Immunopharmacol 23(1) (2014) 37-45.

[71] A. Reinitz, J. DeStefano, M. Ye, A.D. Wong, P.C. Searson, Human brain microvascular endothelial cells resist elongation due to shear stress, Microvascular research 99 (2015) 8-18.

[72] T. Moos, K. Mollgard, Cerebrovascular permeability to azo dyes and plasma proteins in rodents of different ages, Neuropathol Appl Neurobiol 19(2) (1993) 120-7. 